\title{
Workshop Report: USP Workshop on Exploring the Science of Drug Absorption
}

\author{
Marilyn N. Martinez ${ }^{1 *}$, Sid Bhoopathy ${ }^{2}$, Sara Carlert ${ }^{3}$, Murat Cirit ${ }^{4}$, Raafat Fahmy ${ }^{1}$, Talia Flanagan ${ }^{5}$, \\ Ben Forbes ${ }^{6}$, Masoud Jamei ${ }^{7}$, Mansoor A. Khan ${ }^{8}$, Viera Lukacova ${ }^{9}$, Jonathan P. Mochel ${ }^{10}$, Xavier \\ Pepin $^{11}$, Devendra Pade ${ }^{7}$, Christos Reppas ${ }^{12}$, Patrick J. Sinko ${ }^{13}$, David Sperry ${ }^{14}$, Konstantin Tsinman ${ }^{15}$, \\ and Maria Vertzoni ${ }^{12}$ \\ ${ }^{1}$ United States Food and Drug Administration, Center for Veterinary Medicine, Rockville, MD, USA. \\ ${ }^{2}$ Absorption Systems, Exton, PA, USA. \\ ${ }^{3}$ AstraZeneca, Gothenburg, Sweden. \\ ${ }^{4}$ Massachusetts Institute of Technology, Cambridge, MA, USA. \\ ${ }^{5}$ Previously: Research and Development, AstraZeneca, Macclesfield, Cheshire, UK; Present: UCB Pharma SA, Product Development, Chemin du Foriest, \\ Belgium. \\ 'Institute of Pharmaceutical Science, King's College London, London, UK. \\ ${ }^{7}$ Certara UK Limited (Simcyp Division), Sheffield, UK. \\ ${ }^{8}$ Rangel College of Pharmacy, Texas A\&M University, College Station, TX, USA. \\ ${ }^{9}$ Simulation Sciences, Simulations Plus, Inc., Lancaster, CA, USA. \\ ${ }^{10}$ lowa State University College of Veterinary Medicine, Ames, IA, USA. \\ ${ }^{11}$ AstraZeneca, Macclesfield, UK. \\ ${ }^{12}$ Department of Pharmacy, National and Kapodistrian University of Athens, Athens, Greece. \\ ${ }^{13}$ Ernest Mario School of Pharmacy, Rutgers University, The State University of New Jersey, Piscataway, NJ, USA. \\ ${ }^{14}$ Small Molecule Design \& Development, Eli Lilly and Company, Indianapolis, IN, USA. \\ ${ }^{15}$ Pion Inc., Billerica, MA, USA. \\ e-mail: marilyn.martinez@fda.hhs.gov
}

\section{ABSTRACT}

In October 2018, the United States Pharmacopeia (USP) hosted a two-day workshop to explore the science of drug absorption. Experts from around the globe presented some of the challenges associated with drug product development from the perspective of the physiological attributes of the patient (human or canine) and the body site for drug activity. Included in the discussions were methods and approaches for answering complex questions, providing insights into the strengths and challenges of methods available in our biopharmaceutical tool chest. The following is a synopsis of the presentations and the highlights of the discussions that ensued.

Disclaimer: This article reflects the views of the authors and should not be construed as representing the views or policies of the United States Food and Drug Administration.

KEYWORDS: In vitro dissolution, formulation effects, oral drug absorption, interspecies differences, in silico models, in vitro systems, bioavailability, bioequivalence.

\section{INTRODUCTION}

C urrently, the pharmaceutical scientist has an arsenal of in vitro and in silico tools that can be used to assess the in vivo challenges influencing drug product development or to set product quality control (QC) specifications. When used appropriately, these tools can lead to significant time and financial savings while improving the quality of the product available to meet patient therapeutic needs. It can be used to define a "safe space" within which product changes can occur without altering drug product dose-exposure relationships (1).
However, without an appreciation of their limitations and potential sources of bias, these methods can also lead to flawed decisions that negatively influence product quality and availability.

Considering the importance of these tools to both human and veterinary medicine, the United States Pharmacopeia (USP) sponsored a 2-day workshop (October 15-16, 2018), convening international experts to share their experiences and insights across a range of in vitro and in silico tools. The first day focused on oral drug absorption and the translation of factors influencing

*Corresponding author 
product oral bioavailability in humans and dogs. Day 2 provided an opportunity to explore the strengths and weakness of in vitro and in silico systems, focusing on their utility for addressing the evolving challenges facing the development of formulations that meet the needs and expectations of a complex therapeutic landscape. Examples were shared of how these tools have been used to predict drug target site delivery, regardless of whether the active pharmaceutical ingredient (API) is intended for local (e.g., skin, gastrointestinal [GI] tract, or lung) or systemic activity.

The report contains highlights of the workshop presentations and concludes with a summary of the question and answer sessions.

Common abbreviations used in this workshop report include:

- API: active pharmaceutical ingredient

- BA/BE: bioavailability/bioequivalence

- Bcrp or BCRP: breast cancer resistance protein (efflux transporter)

- BCS: Biopharmaceutics Classification System

- BE: bioequivalence

- Caco-2: human colon rectal cancer cell

- CQA: critical quality attribute

- Fa: faction absorbed

- Gl: gastrointestinal

- IVIVC: in vivo-in vitro correlation

- Mdr1 or MDR1: multidrug resistance protein 1 (efflux transporter)

- MDCK: Madin-Darby Canine Kidney Cell

- PBPK model: physiologically based pharmacokinetic model

- QC: quality control

- RLD: reference listed drug product

\section{REFERENCES}

1. Dickinson, P. A.; Lee, W. W.; Stott, P. W.; Townsend, A. I.; Smart, J. P.; Ghahramani, P.; Hammett, T.; Billett, L.; Behn, S.; Gibb, R.C.; Abrahamsson, B. Clinical relevance of dissolution testing in quality by design. AAPS J. 2008, 10, 380-390. DOI: 10.1208/ s12248-008-9034-7.

\section{AIMING FOR THE FUTURE: EXPLORING POSSIBILITIES \\ Marilyn Martinez}

United States Food and Drug Administration, Center for Veterinary Medicine, Rockville, MD, USA.

When formulating an API, an appreciation of the ratelimiting factor in oral bioavailability is essential. For dissolution rate-limited formulations, the drug is absorbed as soon as it dissolves and anything that increases the dissolution rate will likewise increase the amount of drug that gets absorbed. For these drug products, so long as solubility limitation is not an issue, the Fa remains constant and therefore, an increase in administered dose will result in a corresponding increase in the amount of drug absorbed. Similarly, for a permeability rate-limited drug, an increase in dose will lead to a corresponding increase in amount absorbed (i.e., a constant Fa). If the goal is to increase $\mathrm{Fa}$, formulation must be revised in a manner that promotes intestinal permeability. In contrast, for solubility rate-limited drugs, only solubility-enhancing formulations can have a positive impact on the amount of drug absorbed. Without increasing drug solubility, an increase in the administered dose will lead to a decrease in $\mathrm{Fa} \mathrm{(1).}$

An appreciation of what constitutes the rate limiting factor(s) can be supported using in silico models. For example, Chung and Kesisoglou employed a sensitivity analysis to describe the relationship between $\mathrm{Fa}$ and gastric $\mathrm{pH}$ for the weak base, midazolam ( $\mathrm{pKa}=5.5$ and 6.5) (2). Because midazolam is soluble in acid but precipitates in neutral conditions, an increase in the administered dose will lead to a greater dose fraction precipitating (limiting the ability to increase the systemic drug exposure). Similarly, the absorbable dose is highly dependent on the $\mathrm{pH}$ in the Gl tract, resulting in the need to decrease the administered dose in achlorhydric patients.

Understanding formulation CQAs also dictates the in vitro dissolution test conditions necessary to discriminate between inequivalent formulations. He et al. demonstrated that by increasing the amount of surfactant in the dissolution media for a low solubility drug, the dissolution test failed to identify inequivalent formulations (3). This work was used to underscore the importance of having both in vivo and in vitro data across multiple formulations when establishing $\mathrm{QC}$ methods that can assure comparability in the CQAs. 


\section{REFERENCES}

1. Sugano, K.; Okazaki, A.; Sugimoto, S.; Tavornvipas, S.; Omura, A.; Mano, T. Solubility and dissolution profile assessment in drug discovery. Drug Metab. Pharmacokinet. 2007, 22, 225-254. DOI: 10.2133/dmpk.22.225.

2. Chung, J.; Kesisoglou, F. Physiologically based oral absorption modelling to study gut-level drug interactions. J. Pharm. Sci. 2018, 107, 18-23. DOI: 10.1016/j.xphs.2017.08.015.

3. He, Z; Zhong, D.; Chen, X.; Liu, X.; Tang, X.; Zhao, L. Development of a dissolution medium for nimodipine tablets based on bioavailability evaluation. Eur. J. Pharm. Sci. 2004, 21, 487-491. DOI: 10.1016/j.ejps.2003.11.009.

\section{EXAMPLES OF DISSOLUTION DIFFERENCES WHEN USING CANINE VS. HUMAN BIORELEVANT MEDIA: WORKING TO OPTIMIZE IN VITRO METHODS THAT SUPPORT THE TRANSLATION OF IN VIVO ORAL DRUG PRODUCT DISSOLUTION IN DOGS VS. HUMANS Maria Vertzoni}

Department of Pharmacy, National and Kapodistrian University of Athens, Athens, Greece.

The use of biorelevant media for the evaluation of dosage form performance within the Gl lumen of Caucasian adults was first introduced in the late 1990s (1). Since then, our knowledge of the human Gl environment has improved, and a variety of additional media have been proposed (Figure 1) (2). The type of information derived from these studies depends upon the complexity of the media (referred to a "Level" in Figure 1), and the Level of simulation necessary depends on the information one is seeking, the characteristics of the API, and the dosage form. A simulation of all aspects of luminal conditions may or may not be necessary to evaluate drug product performance (3).

When developing canine therapeutics, the physiological diversity resulting from selective breeding and the largely uncontrolled dosing conditions can lead to wide variability in oral drug product performance. This renders the development of biorelevant media more difficult to define. To date, only media for the simulation of the fasted canine upper $\mathrm{Gl}$ environment have been proposed (4).

The usefulness of human and canine biorelevant media to estimate corresponding drug solubilities has been illustrated with case examples (4-7). The interspecies differences in gastric and intestinal $\mathrm{pH}$ of humans and dogs can influence the solubility of lipophilic APIs with
pH-dependent solubility (i.e., weak acids and weak bases), and differences in the composition and concentration of bile salts may have an impact on drug solubility, especially for APIs with high logP values.

\section{Levels of simulation of luminal composition}

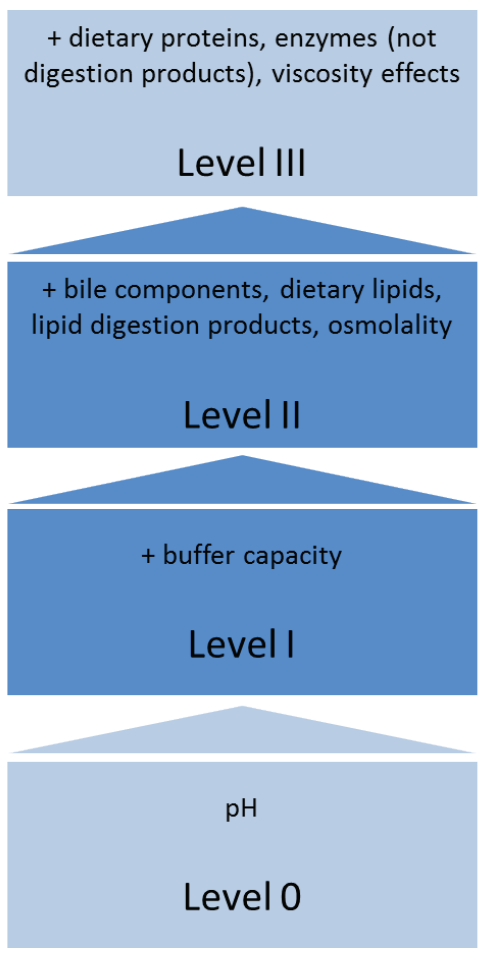

Figure 1. An overview of the four levels of biorelevant media recommended for the simulation of the luminal environment during development of oral formulations.

Reprinted with permission from Markopoulos, C.; Andreas, C.; Vertzoni, M.; Dressman, J.; Reppas, C. In-vitro simulation of luminal conditions for evaluation of performance of oral drug products: choosing the appropriate test media. Eur. J. Pharm. Biopharm. 2015, 93, 173-182. Copyright 2015 American Chemical Society.

Biorelevant media may also facilitate a prediction of oral absorption. For example, dissolution data generated under conditions simulating the human upper and lower intestine were used to simulate either the average plasma levels or the average absorption process (8). It was concluded that luminal dissolution kinetics, estimated by using compendial dissolution setups and human biorelevant media, in combination with PBPK modelling approaches, can be useful for assessing the effect of formulation and food on drug blood level profiles in healthy adults (Figure 2).

Regarding the evaluation of the predictability of oral absorption in dogs using canine biorelevant media, it was pointed out that clarification of reference dosing 
conditions in dogs and information on the luminal conditions under these conditions (especially in the fed state) are needed for optimizing and/or proposing new canine biorelevant media. Similarly, PBPK modeling data with various APIs/products are needed to confirm the usefulness of biorelevant media in modelling oral drug absorption in dogs.
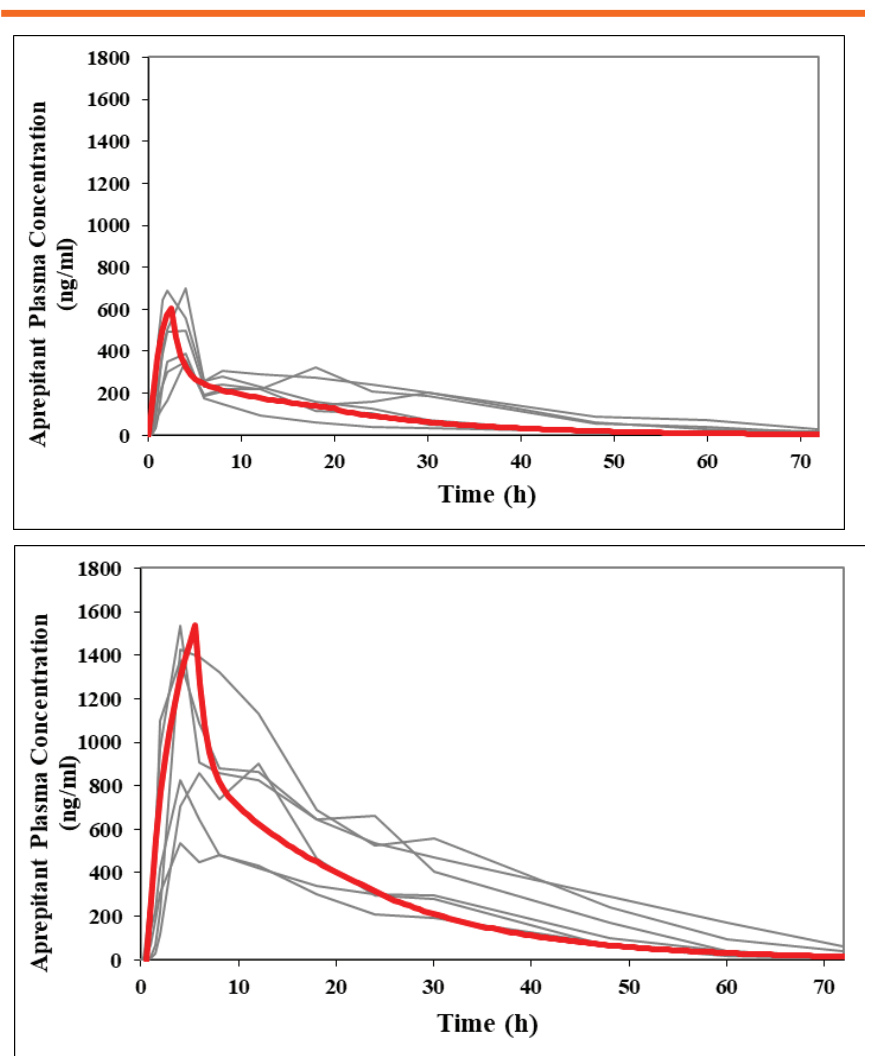

Figure 2. Individual measured plasma concentrations vs time after single dose administration of $100 \mathrm{mg}$ of micronized aprepitant to healthy fasted adults (gray lines) and simulated plasma profiles (bold red line) in the fasted state (A) and in the fed state (B) using data in Level II biorelevant media coupled with PBPK modeling. Simulations were performed using a physiologically based oral absorption model for passively absorbed APIs and STELLA 9.0.2 software (Isee Systems, Inc., Lebanon, NH, USA). Reprinted with permission from Mol. Pharm., Vol. 14, Georgaka, D.; Butler, J.; Kesisoglou, F.; Reppas, C.; Vertzoni, M. Evaluation of dissolution in the lower intestine and its impact on the absorption process of high dose low solubility drugs, 4181-4191, Copyright 2017, with permission from Elsevier.

\section{REFERENCES}

1. Dressman, J.B.; Amidon, G.L.; Reppas, C.; Shah, V.P. Dissolution testing as a prognostic tool for oral drug absorption: immediate release dosage forms. Pharm. Res. 1998, 15, 11-22. DOI: 10.1023/A:1011984216775.

2. Markopoulos, C.; Andreas, C.; Vertzoni, M.; Dressman, J.; Reppas, C. In-vitro simulation of luminal conditions for evaluation of performance of oral drug products: choosing the appropriate test media. Eur. J. Pharm. Biopharm. 2015, 93, 173-182. DOI:10.1016/j.ejpb.2015.03.009.

3. Friedel, H.D.; Brown, C.K.; Barker, A.R.; Buhse, L.F.; Keitel, S.; Kraemer, J.; Morris, J. M.; Reppas, C.; Sperry, D. C.; Sakai-Kato,
K.; Stickelmeyer, M. P.; Shah, V. P. FIP Guidelines for Dissolution Testing of Solid Oral Products. J. Pharm. Sci. 2018, 107, 29953002. DOI:10.1016/j.xphs.2018.08.007.

4. Arndt, M.; Chokshi, H.; Tang, K.; Parrott, N.J.; Reppas, C.; Dressman, J. B. Dissolution media simulating the proximal canine gastrointestinal tract in the fasted state. Eur. J. Pharm. Biopharm. 2013, 84, 633-641. DOI: 10.1016/j.ejpb.2013.01.010.

5. Kalantzi, L.; Persson, E.; Polentarutti, B.; Abrahamsson, B.; Goumas, K.; Dressman, J. B.; Reppas, C. Canine intestinal contents vs. simulated media for the assessment of solubility of two weak bases in the human small intestinal contents. Pharm. Res. 2006, 23, 1373-1381. DOI: 10.1007/s11095-006-0207-8.

6. Vertzoni, M.; Diakidou, A.; Chatzilias, M.; Söderlind, E.; Abrahamsson, B.; Dressman, J. B.; Reppas, C. Biorelevant media to simulate fluids in the ascending colon of humans and their usefulness in predicting intracolonic drug solubility. Pharm. Res. 2010, 27, 2187-2196. DOI: 10.1007/s11095-010-0223-6.

7. Vertzoni, M.; Pastelli, E.; Psachoulias, D.; Kalantzi, L.; Reppas, C. Estimation of intragastric solubility of drugs: In what medium? Pharm. Res. 2007, 24, 909-917. DOI: 10.1007/s11095-0069209-9.

8. Georgaka, D.; Butler, J.; Kesisoglou, F.; Reppas, C.; Vertzoni, M. Evaluation of dissolution in the lower intestine and its impact on the absorption process of high dose low solubility drugs. Mol. Pharm. 2017, 14, 4181-4191. DOI: 10.1021/acs. molpharmaceut.6b01129.

CHARACTERIZATION OF GI FLUIDS CONTENT, VISCOSITY, VOLUME IN DOGS AND HUMANS: COMPARISON UNDER FASTED AND FED STATE

\section{CONDITIONS}

\section{Christos Reppas}

Department of Pharmacy, National and Kapodistrian University of Athens, Athens, Greece.

Human data have typically been collected either after an overnight fast along with a glass of water (fasted state) or following a standard solid meal (fed state) $(1,2)$.

In this presentation, the Gl tract conditions of dogs and humans in fed and fasted states were compared. If information after the standard meal does not exist, data from liquid meals or other solid meals having similar composition to that of the standard meal were considered. Canine data were collected in L abradors, mongrels ( $\sim 25$ $\mathrm{kg})$, and/or Beagles either after an overnight fast with the administration of varying volumes of water (fasted state), or after an overnight fast with subsequent administration of either (a portion of) the standard meal or dog food (fed state). Due to substantial differences between the composition of the standard human meal $(1,2)$ versus that 
of dog food (3), two different fed states were considered in dogs.

Fasted state: Although the canine gastric $\mathrm{pH}$ is acidic, it is typically higher and more variable than that of humans (e.g., 4). In the upper intestine (duodenum and jejunum) the $\mathrm{pH}$ is slightly higher than in humans $(4,5)$. The canine lower intestine (distal ileum and ascending colon) is slightly more alkaline than that of humans (5). Species differences in buffer capacity, osmolality, surface tension and esterase activity in upper intestine seem to be nonsignificant $(5,6,7)$. However, bile salt content is higher in the canine upper intestine (4). In the lower intestine, relevant data have been published only for humans (5). Species differences in the viscosity of contents of upper gastrointestinal lumen seem to be minimal, with values being slightly higher than an aqueous solution $(5,8)$. In humans, volumes of gastric contents at rest, of duodenal contents and of contents of ascending colon are variable but roughly about $25 \mathrm{~mL}$ in each of these regions $(5,9)$. In dogs, although published data are few, the resting gastric resting volumes in dogs and humans appear to be similar (10).

Fed state: The canine gastric $\mathrm{pH}$ is acidic after dog food (e.g., 3). After consuming a (body weight normalized amount of) standard human meal, the $\mathrm{pH}$ is similar to that of humans (11), i.e., less acidic than in the fasted state or after dog food. In the upper intestine of dogs and humans, data after administration of liquid meals indicate slightly decreased $\mathrm{pH}$ as compared with the values observed in the fasted state $(5,7)$. After administration of a solid meal, $\mathrm{pH}$ was reduced the in mid-jejunum of mongrels (8). No data are available after dog food or after administration of the standard meal to humans. In distal ileum, the $\mathrm{pH}$ is not affected by the administration of the standard meal to humans but in the ascending colon it is decreased significantly to slightly acidic values (5). No data are published on the dog lower intestine. After administration of "human" meals, the upper intestine buffer capacity and osmolality are higher in dogs that in humans whereas surface tension is lower in dogs than in humans (e.g., 5, 7, 8). There are no corresponding data after dog food. In the human lower intestine, buffer capacity, and osmolality are increased after administration of the standard meal whereas surface tension is decreased (5). There are no corresponding data in dogs. Viscosity in mid-jejunum of dogs after administration of a solid meal similar with the standard meal is variable but not substantially different than in the fasted state (8). There are no data in humans. Intragastric volumes after the standard meal to humans is about $600 \mathrm{~mL}$ during the first $1-1.5$ hours post meal administration. Two hours after administration of a similar solid meal to humans, about $1.5 \mathrm{~L}$ of fluid pass from the duodenum and about $0.75 \mathrm{~L}$ pass from proximal jejunum (5). There are no relevant data in dogs. Intraluminal volumes in the lower intestine are only slightly affected by the administration of the standard meal to humans (5). No corresponding information is available for dogs.

\section{REFERENCES}

1. Food-effect bioavailability and fed bioequivalence studies. Guidance for Industry: U.S. Department of Health and Huamn Services, Food and Drug Administration, Center for Drug Evaluation and Research (CDER), Silver Spring MD: 2002.

2. Guideline on the investigation of bioequivalence. European Medicines Agency, London UK; 2010.

3. Song, Y.; Peressin, K.; Wong, P.Y.; Page, S.; Carg, S. Key Considerations in designing oral drug delivery systems for dogs J. Pharm Sci 2016, 105, 1576-1585. DOI: 10.1016/j. xphs.2016.03.007.

4. Arndt, M.; Chokshi, H.; Tang, K.; Parrott, N.J,; Reppas, C.; Dressman, J. B. Dissolution media simulating the proximal canine gastrointestinal tract in the fasted state. Eur. J. Pharm. Biopharm. 2013, 84, 633-641. DOI: 10.1016/j.ejpb.2013.01.010.

5. Pentafragka, C.; Symillides, M.; McAllister, M.; Dressman, J.; Vertzoni, M.; Reppas, C. The impact of food intake on the luminal environment and performance of oral drug products with a view to in vitro and in silico simulations: a PEARRL review. J. Pharm. Pharmacol. 2019, 71, 557-580. DOI: 10.1111/jphp.12999.

6. Borde, A.S.; Karlsson, E.M.; Andersson, K.; Björhall, K.; Lennernäs, H.; Abrahamsson, B. Assessment of enzymatic prodrug stability in human, dog and simulated intestinal fluids. Eur. J. Pharm. Biopharm. 2012, 80, 630-637. DOI: 10.1016/j.ejpb.2011.11.011.

7. Kalantzi, L.; Persson, E.; Polentarutti, B.; Abrahamsson, B.; Goumas, K.; Dressman, J. B.; Reppas, C. Canine intestinal contents vs. simulated media for the assessment of solubility of two weak bases in the human small intestinal contents. Pharm. Res. 2006, 23, 1373-1381. DOI: 10.1007/s11095-006-0207-8.

8. Greenwood, D. E. Small intestinal $\mathrm{pH}$ and buffer capacity: implications for dissolution of ionizable compounds. PhD Thesis, The University of Michigan, 1994.

9. Grimm, M.; Koziolek, M.; Kühn, J. P.; Weitschies, W. Interindividual and intraindividual variability of fasted state gastric fluid volume and gastric emptying of water. Eur. J. Pharm. Biopharm. 2018, 127, 309-317. DOI: 10.1016/j.ejpb.2018.03.002.

10. Lentner, C. Geigy Scientific Tables, In: Units of measurement, body fluids, composition of the body, nutrition. Vol 1. Basel Switzerland: Ciba-Geigy; 1981.

11. Koziolek, M.; Grimm, M.; Bollmann, T.; Schäfer, K.J.; Blattner, S.M.; Lotz, R.; Boeck, G.; Weitschies, W. Characterization of the GI transit conditions in Beagle dogs with a telemetric motility capsule. Eur. J. Pharm. Biopharm. 2019, 136, 221-230. DOI: 10.1016/j.ejpb.2019.01.026. 
IN-SILICO MODELLING OF ORAL DRUG

\section{ABSORPTION - DOGS VS HUMANS: DIFFERENCES IN GI TRACT ANATOMY \& PHYSIOLOGY Devendra Pade}

Certara UK Limited (Simcyp Division), Blades Enterprise Center, Sheffield, UK.

A clear understanding of the anatomy and physiology of the GI tract is essential for a mechanistic understanding of the in vivo process of oral drug absorption (1). Accordingly, when using the beagle dog as a biopharmaceutical model for extrapolating orally administered formulations to humans, one should be aware of the Gl tract differences between these two species.

PBPK models factor the species-specific physiology and its role in influencing the intestinal behavior of a drug to help understand, verify and extrapolate oral absorption characteristics from one species to another. Dividing the model into 'system' (species) and 'drug' parameters helps identify critical parameters with respect to species physiology and/or drug-formulation. A robust model that passes the initial steps of performance verification in a particular species may fail during extrapolation to the human (as a 'false negative') if system parameters are not robust and if the modeler lacks awareness of the critical parameters and their differences between the two species. Table 1 below highlights the primary anatomical and physiological differences between the dog and human GI tract as incorporated within the Simcyp Simulator ${ }^{\circledast}$ (Version 17).

As seen from Table 1, there are no major humancanine differences in some parameters such as gastric emptying, small intestinal (SI) transit time and bile salt concentrations. However, major differences exist for other parameters and these differences can profoundly impact the absorption of a drug after oral administration. An example of the impact of such differences can be seen when using the MechPeff model (2) which can predict the passive intestinal permeability of a drug after taking into consideration the anatomy (such as villus morphology), physiology (such as $\mathrm{pH}$, bile salts concentration etc.) and the physicochemical properties of the drug. A comprehensive verification of this model in different species has been published earlier $(3,4)$.

Although complex in silico mechanistic models can be developed to predict drug PK in human and preclinical species, it is important to populate such models with robust system and drug parameters. If a complex model is populated with data arising from flawed assumptions, this can lead to potential false positive or negative performance verifications. Finally, the modeler should be aware of the primary differences between the 'systems' (i.e., physiologies) being modelled, and a sensitivity analysis should help support assumptions pertaining to the CQAs as a function of the targeted species.

Table 1. Mean Anatomy and Physiology Parameters Incorporated Within the Simcyp Human and Beagle Dog simulator (Version 17).

\begin{tabular}{|c|c|c|c|c|c|c|c|c|c|}
\hline \multirow[b]{2}{*}{ Simcyp } & \multirow{2}{*}{$\begin{array}{c}\text { SI Length/ } \\
\text { Diameter }(\mathrm{cm})\end{array}$} & \multicolumn{3}{|c|}{ Transit Time (h) } & \multirow{2}{*}{$\begin{array}{l}\text { Bile (mM) } \\
\text { Jejunum }\end{array}$} & \multicolumn{2}{|c|}{ pH (fasted) } & \multirow{2}{*}{$\begin{array}{c}\begin{array}{c}\text { Secretion } \\
(\mathrm{mL} / \mathrm{h})\end{array} \\
\text { Duodenum }\end{array}$} & \multirow{2}{*}{$\begin{array}{c}\text { CYP3A } \\
\text { (nmol) }\end{array}$} \\
\hline & & Gastric & SI & Colon & & Gastric & SI & & \\
\hline Dog & $262 / 1$ & 0.37 & 2.4 & 7.5 & 5 & $1-8$ & $6.1-6.8$ & 27 & 44 \\
\hline Human & $723 / 4$ & 0.4 & 3.3 & 12 & $2-4$ & $1.2-2.5$ & $6.4-7.4$ & 89 & 68 \\
\hline \multirow[b]{2}{*}{ Simcyp } & \multirow{2}{*}{\multicolumn{2}{|c|}{ Intestinal Transporter }} & \multicolumn{5}{|c|}{ Intestinal Transporter Distribution } & & \\
\hline & & & \multicolumn{2}{|c|}{ Duodenum } & $\begin{array}{c}\text { Mid-Distal } \\
\text { Jejunum }\end{array}$ & Ileum & Colon & \multirow{3}{*}{\multicolumn{2}{|c|}{$\begin{array}{l}\text { *Generic transporter; mRNA } \\
\text { data (1) } \\
\wedge \text { Transporter distribution } \\
\text { relative to proximal Jejunum }\end{array}$}} \\
\hline Dog* & \multicolumn{2}{|l|}{$\begin{array}{l}\text { Mdr1 } \\
\text { Bcrp }\end{array}$} & \multicolumn{2}{|c|}{$\begin{array}{c}0.41 \\
0.1\end{array}$} & $\begin{array}{c}0.53-1 \\
0.11\end{array}$ & $\begin{array}{c}0.9-1 \\
0.07\end{array}$ & $\begin{array}{l}0.61 \\
0.03\end{array}$ & & \\
\hline Human^ & \multicolumn{2}{|l|}{$\begin{array}{l}\text { MDR1 } \\
\text { BCRP }\end{array}$} & \multicolumn{2}{|c|}{$\begin{array}{l}0.51 \\
0.47\end{array}$} & $\begin{array}{c}1-1.5 \\
1\end{array}$ & $\begin{array}{c}1.46 \\
0.6\end{array}$ & $\begin{array}{l}0.6 \\
0.1\end{array}$ & & \\
\hline \multirow[t]{2}{*}{ Simcyp } & \multicolumn{2}{|c|}{ Villus Shape } & \multicolumn{5}{|c|}{ Villus Height $(\mu \mathrm{m})$} & \multirow{2}{*}{\multicolumn{2}{|c|}{ Folds of the Plicae }} \\
\hline & & & \multicolumn{2}{|c|}{ Duodenum } & Jejunum & Ileum & Colon & & \\
\hline Dog & \multicolumn{2}{|c|}{ Cylindrical } & \multicolumn{2}{|c|}{935} & 718 & 527 & \multirow{2}{*}{ No villi } & \multicolumn{2}{|c|}{ Absent } \\
\hline Human & \multicolumn{2}{|c|}{ Leaf shaped } & \multicolumn{2}{|c|}{523} & 449 & 290 & & & \\
\hline
\end{tabular}




\section{REFERENCES}

1. Haller, S.; Schuler, F.; Lazic, S.E.; Bachir-Cherif, D.; Kramer, S.D.; Parrott, N.J.; Steiner, G.; Belli S. Expression profiles of metabolic enzymes and drug transporters in the liver and along the intestine of beagle dogs. Drug Metab. Dispos. 2012, 40, 1603-1610. DOI: 10.1124/dmd.112.045443.

2. Pade, D.; Jamei, M.; Rostami-Hodjegan, A.; Turner, D. B. Application of the MechPeff model to predict passive effective intestinal permeability in the different regions of the rodent small intestine and colon. Biopharm. Drug Dispos. 2017, 38, 94-114. DOI: 10.1002/bdd.2072.

3. Pade, D.; Jamei, M.; Rostami-Hodjegan, A.; Turner, D. B. A mechanistic framework for the in silico prediction of regional passive gut wall permeability and its inter-individual variability in humans. Presented at the 2014 Annual Meeting of the American Association of Pharmaceutical Scientists. San Diego, CA, USA.

4. Pade, D.; Jamei, M.; Salem, F.; Gill, K.; Turner, D. B. In silico prediction of regional passive intestinal permeability in cynomolgus monkey using 'MechPeff': a mechanistic model with drug physicochemical and in vitro parameters as inputs. Presented at the 2015 Annual Meeting of the American Association of Pharmaceutical Scientists. Orlando, FL, USA Poster W5029.

\section{COMPARISON OF PERMEABILITY WITH DATA} GENERATED IN USSING CHAMBER (HUMAN, DOG, RAT), INTESTINAL PERFUSION, MDCK AND

\section{CACO-2 CELLS}

Sid Bhoopathy

Absorption Systems, Exton, PA, USA.

Permeability is an important property of a drug substance as it helps guide various aspects of drug product development. These include lead optimization, formulation development, predicting drug disposition, understanding food effects and obtaining BCS-based biowaivers.

Multiple non-clinical techniques are available for its determination. Of these, cell monolayers (such as those comprised of Caco-2 and MDCK cells) in a transwell format are the most prevalent. To achieve reliable and reproducible results, it is essential that certain controls be instituted. These include the use of appropriate cell culture analytics, limitations on culture age (as defined by passage number and days-in-culture), pre-determined QC criteria, use of model compound(s) co-dosed with a test drug to rank order its permeability, and the assessment of post-experimental monolayer integrity. Other experimental considerations include the determination of drug non-specific binding to the apparatus (this problem can be alleviated by using a pre-incubation of the test compound on the donor side and bovine serum albumin fortified receptor solution on the receiver side), and the use of multiple receiver time points for calculating the linear portion of the permeability-time profile. The use of excipients to improve solubility on either the donor or the receiver sides is acceptable, if these solubility enhancing agents have been previously shown to be tolerated by the cells.

Other techniques for permeability determination are intestinal perfusion and the excised intestinal tissue model. The intestinal perfusion model can be in a closed-loop, single pass or a recirculating format. The Ussing chamber (e.g., an intestinal tissue model) can be run with either preclinical or human tissue. Experimental considerations are similar to that of cell-based models.

Each of these models was correlated independently to human Fa by testing several model compounds. Additionally, a sub-set of the compounds were run in the Caco-2, MDCK, dog jejunum Ussing chamber, ratjejunum Ussing chamber and rat single pass intestinal perfusion models. These compounds were: antipyrine and minoxidil, both associated with a high fraction absorbed ( $\geq 85 \%$ ); the moderately absorbed drugs, amiloride, hydrochlorothiazide, and atenolol ( $\mathrm{Fa}=50$ to $84 \%)$; and the poorly absorbed drugs, lisinopril and FD-4 (Fa < 50\%). Caco-2 and MDCK cells had the largest dynamic range of permeability values (over two orders of magnitude) and demonstrated the greatest discrimination among the various $\mathrm{Fa}$ categories. Rat intestinal perfusion and rat intestinal tissue models had the correct Fa-category rank order for a sub-set of seven compounds but exhibited a smaller dynamic range as compared to that of the cell monolayers. Tissue based techniques exhibit a high degree of covariance and therefore, test compounds need to be co-dosed with a high and low permeability internal standard to compute the normalized test drug permeability. The dog model did not correlate with the human Fa or with the other permeation techniques for the test drugs examined. Data from additional donors and revised tissue manipulation techniques maybe reviewed to improve outcomes.

Each of these models was correlated independently to human Fa by testing several model compounds. Additionally, a sub-set of the compounds was run in the Caco-2, MDCK, dog jejunum Ussing chamber, ratjejunum Ussing chamber and rat single pass intestinal perfusion models. These compounds were: antipyrine and minoxidil, both associated with a high fraction 
absorbed ( $\mathrm{Fa} \geq 85 \%$ ); the moderately absorbed drugs, amiloride, hydrochlorothiazide, and atenolol (Fa between 50 to $84 \%$ ); and the poorly absorbed drugs, lisinopril and FD-4 (Fa < 50\%). Caco-2 and MDCK cells had the largest dynamic range of permeability values (over two orders of magnitude) and demonstrated the greatest discrimination among the various Fa categories. Rat intestinal perfusion and rat intestinal tissue models had the correct Facategory vs. permeation rank order for this sub-set of seven compounds but exhibited a smaller dynamic range as compared to that of the cell monolayers. Tissue based techniques exhibit a high degree of covariance and therefore, test compounds need to be co-dosed with a high and low permeability internal standard to compute the normalized test drug permeability. The dog model did not correlate with the human Fa or with the other permeation techniques for the test drugs examined. Data from additional donors and revised tissue manipulation techniques may be reviewed to improve outcomes.

A combined dissolution and absorption system that can simultaneously quantify both processes (Figure. 1) is currently under development to improve the IVIVC of in vitro product performance tools and to assess the interplay of drug release and permeation $(1,2)$.

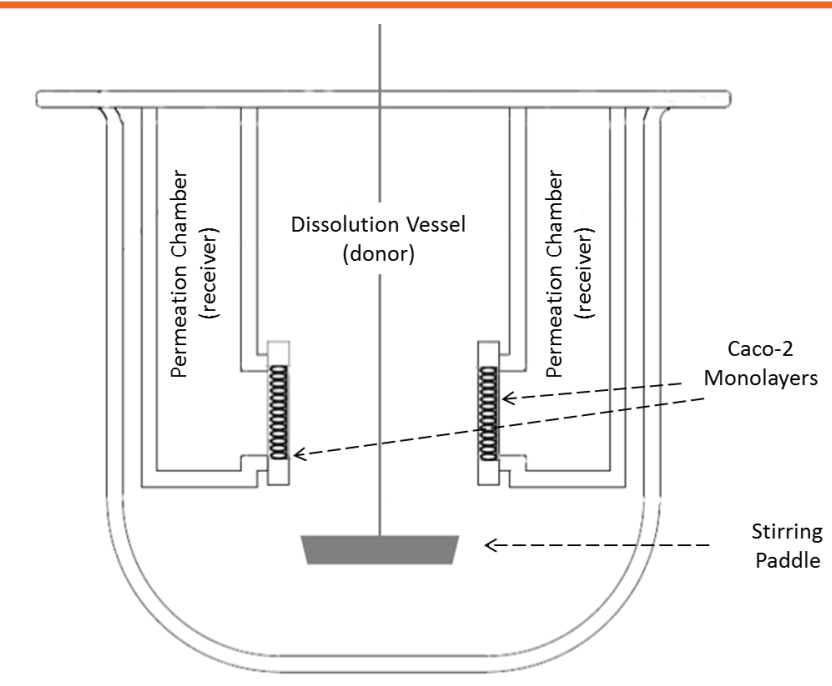

Figure 1. In Vitro Dissolution Absorption System (IDAS)

\section{REFERENCES}

1. Murray, L.; Arias, A.; Li, J.; Bhoopathy, S.; Hidalgo, I.J. Innovative in vitro methodologies for establishing therapeutic equivalence. Rev Panam Salud Publica. 2016, 40, 23-28.

2. Li, J.; Li, L.B.; Nessah. N.; Huang. Y.; Hidalgo, C.; Owen, A.; Hidalgo, I. J. Simultaneous analysis of dissolution and permeation profiles of nanosized and microsized formulations of indomethacin using the in vitro dissolution absorption system 2. J. Pharm. Sci. 2019, 108, 2334-2340.

\section{CANINE ORGANOIDS FOR DRUG TESTING: MOVING BEYOND CACO-2 CELL SYSTEMS Jonathan P. Mochel}

lowa State University, College of Veterinary Medicine, Ames, IA, USA.

In its white paper on New Medical Technologies, the US FDA emphasized the need to improve the predictability and efficiency of studies impacting drug product development (1). While methods are available for evaluating drugintestinal cross-talk for human therapeutics, there is little corresponding information currently available for dogs. There is, therefore, a critical need to develop new methods for studying drug-gut interactions using scalable and physiological platforms in dogs.

Stem cells-derived 3-dimensional (3D) tissue models termed Organoids were awarded Nature Methods of the Year status in 2017 (2). Organoids present multiple advantages over standard in vitro models, including cancer-derived cell lines (e.g. Caco-2, T84, and HT29), or spontaneously immortalized epithelial cells (e.g. Rat Intestinal Epithelial (RIE) cultures) (3). These include the method's ability to recapitulate in vivo cellular phenotypes and the opportunity to collect biological samples from individuals with different genotypes, environmental risk factors (e.g., diet, microbiota) and drug sensitivity profiles. The development of a microphysiological gut system that morphologically, biologically and structurally replicates the endogenous epithelium shows tremendous potential to evaluate the transport and intestinal metabolism of drugs administered through the oral route.

Within the framework of a 5-year research collaboration agreement with the FDA (4), our laboratory has generated key preliminary data for the phenotypical characterization of canine intestinal organoids $(5,6)$. Ten endoscopic biopsies of intestinal (duodenum, jejunum, ileum and colon) mucosa from 31 healthy dogs and 23 dogs with chronic enteropathies were collected for crypt isolation. The adult stem cells were then cultured using Intestinal Stem Cell (ISC) growth media. Optimized ISC growth media contained rho-associated kinase inhibitor Y27632, glycogen synthase kinase $3 \beta$ inhibitor CHIR99021, and wnt-3a. Organoids were passaged weekly over 9 months. Characterization of organoids was performed using brightfield imaging, transmission electron microscopy, immunohistochemistry and RNA in situ hybridization for select cell markers, including: (1) intestinal stem cells 
(LGR5/SOX9); (2) absorptive enterocytes (ALP1/IL-15); (3) enteroendocrine cells (NeuroG3); (4) Goblet cells (MUC2); and (5) Tuft chemosensory cells (DCLK1). Our results showed that organoids from primarily stem cell spheroids (Day 3) can differentiate into enterocytes, Goblet cells, enteroendocrine cells, Paneth-like cells and Tuft cells mosaics (Day 6-Day 8). Ultrastructural characterization confirmed increasing density of tight junction proteins from Day 1 to Day 9. Functional assays, using LPS stimulation, showed increased growth rate of intestinal organoids, as well as differential mRNA gene expression profiles, as reported in vivo in the context of chronic intestinal inflammatory diseases.

These preliminary findings support the development of a novel and translatable animal model for the in vitro characterization of drug transport and metabolism as a function of intestinal segment (from duodenum to colon) and disease. Upon completion, this research is expected to provide the animal health community with an appreciation of factors that impact intestinal absorption of drugs intended for use in companion animals. This understanding will be important to support the evaluation of product formulation and in vivo dissolution for oral dosage forms. Ultimately, data generated through this research program are anticipated to improve the utility of in silico models for better characterizing canine drugdose-exposure-response relationship (7). Altogether, the results generated by these studies will provide invaluable information to assist Drug Sponsors for the interpretation of oral bioavailability data and its influence on drug efficacy and safety in field clinical trials for parallel drug development in animal and human health (8). Equally important, these results will have a positive impact by determining how chronic inflammation can affect drug permeability and gut metabolism, thereby supporting the therapeutic decisions of veterinary practitioners.

\section{REFERENCES}

1. US Food and Drug Administration. Innovation or Stagnation: Challenge and Opportunity on the Critical Path to New Medical Products. 2004. Available from:https://www.fda.gov/ ScienceResearch/SpecialTopics/CriticalPathInitiative/default.htm.

2. Method of the Year 2017: Organoids. Nature Methods. 2018, 15. DOI 10.1038/nmeth.4575.

3. Sun, D.; Yu, L. X.; Hussain, M. A.; Wall, D. A.; Smith, R. L.; Amidon, G. L. In vitro testing of drug absorption for drug 'developability' assessment: forming an interface between in vitro preclinical data and clinical outcome. Curr. Opin. Drug Discov. Devel. 2004, 7, 75-85.

4. HHS-Food and Drug Administration (FDA) Research Collaboration Agreement 019765-00001 (10/11/2018-10/10/2023).
5. Mochel, J. P..; Jergens, A. E.; Kingsbury, D.; Kim, H. J.; Martín, M. G.; Allenspach, K. Intestinal stem cells to advance drug development, precision, and regenerative medicine: a paradigm shift in translational research. AAPS J. 2017, 20,17. DOI: 10.1208/ s12248-017-0178-1.

6. Chandra, L.; Borcherding, D. C.; Kingsbury, D.; Atherly, T.; Ambrosini, Y. M.; Bourgois-Mochel, A.; Yuan. W.; Kimber, M.; Qi, Y.; Wang, Q.; Wannemuehler, M.; Ellinwood, N. M.; Snella, E.; Martin, M.; Skala, M.; Meyerholz, D.; Estes, M.; FernandezZapico, M. E.; Jergens, A. E.; Mochel, J. P.; Allenspach K. Derivation of adult canine intestinal organoids for translational research in gastroenterology. BMC Biol. 2019, 17, 33. DOI: 10.1186/s12915019-0652-6.

7. Bon, C.; Toutain, P. L.; Concordet, D.; Gehring, R.; Martin-Jimenez, T.; Smith, J.; Pelligand, L.; Martinez, M.; Whittem, T.; Riviere, J. E.; Mochel, J. P. Mathematical modeling and simulation in animal health. Part III: Using nonlinear mixed-effects to characterize and quantify variability in drug pharmacokinetics. J Vet Pharmacol Ther. 2018, 41, 171-183. DOI: 10.1111/jvp.12473.

8. Schneider, B.; Balbas-Martinez, V.; Jergens, A. E.; Troconiz, I. F.; Allenspach, K.; Mochel, J. P. Model-based reverse translation between veterinary and human medicine: the one health initiative. CPT Pharmacometrics Syst Pharmacol. 2018, 7, 65-68. DOI: 10.1002/psp4.12262.

\section{USE OF THE DOG TO PREDICT SUPERSATURATION AND ITS IMPACT ON DRUG ABSORPTION \\ Sara Carlert}

AstraZeneca, Gothenburg, Sweden.

Quantifying in vivo effects of supersaturation and precipitation of poorly soluble drugs has been a challenging goal, mainly due to the difficulty of measuring concentrations and determining solid state forms of the precipitating drug directly in the stomach or the intestines. Solubility, precipitation rate and redissolution rate can be highly dependent on the physical form of the precipitate, leading to difficulty in interpreting and correlating in vitro and in vivo precipitation investigations. Simple in vitro experiments have been shown to overestimate in vivo precipitation. There have been reports of successful attempts at combining in vitro experiments with in silico predictions, as well as reports on more complex in vitro experimental setups with absorption compartments that have described in vivo intestinal concentrations. But there are still very few examples of intestinal in vivo concentration data of compounds where precipitation has had a significant effect on in vivo intestinal concentrations and subsequent bioavailability. 
In the process of developing a new drug, precipitation investigations must always be put into context. As precipitation rate is concentration dependent, factors such as clinical dose range, margins of safety, therapeutic dose and limitation in acceptance for variability could affect the effort put into understanding the risks of in vivo precipitation. The drive for reducing the use of animals in pharmaceutical development has led to more sophisticated in vitro and in silico tools, but risk assessment of potential discrepancies between in vitro and in vivo behaviour is still key, where pre-clinical species are often used to de-risk clinical failures.

Development and validation of in vitro and in silico models can benefit from pre-clinical in vivo models, but the choice of animal is important for human correlation. One of the more promising models are large dogs. Large dogs are more similar to humans than are small dogs and therefore are recommended to be used for supersaturation and precipitation investigations. However, even when using large dogs, there are several interspecies differences that can affect the interpretation of study results: 1) The gastric $\mathrm{pH}$ of fasted dogs is usually more variable than that of humans, which could affect the level of supersaturation achievable in vivo. This variability can be reduced by administering acidic fluids. However, such an adjustment could alter gastric emptying rate (1); 2) Motility is another factor to consider, where that of large dogs tend to be similar to that observed in humans; 3) Nevertheless, dogs generally have faster GI transit of contents as compared to that of humans. Presumably, this is at least partially due to dog-human differences in body size (2). Faster transit can result in less time for drug precipitation and redissolution; 4) Permeability is also a key factor for understanding precipitation, as it directly affects the intestinal concentration and supersaturation of drug. Canine intestinal permeability tends to be higher than that of humans for low permeability compounds and may also be higher for some high permeability drugs (2, 3); 5) The extent of supersaturation can also be affected by the comparatively low volume of fluids administered to dogs as compared to humans; 6) Dogs present a risk of vomiting and coprophagia, both which render precipitation investigations difficult to interpret.

Four cases investigating the levels and impact of supersaturation and precipitation were discussed. By knowing the limitations of the models and understanding the drug compounds studied, dogs have been successfully used to assess supersaturation and to verify in vivo precipitation effects $(4,5)$ (either directly in the intestines or through a combination of in vitro and in silico models).
Dogs have also been used as a tool for selecting a formulation or solid-state form by aiding predictions, particularly when no IVIVC has been established (6).

\section{REFERENCES}

1. Polentarutti, B.; Albery, T.; Dressman, J.; Abrahamsson, B. Modification of gastric $\mathrm{pH}$ in the fasted dog. J. Pharm. Pharmacol. 2010, 62, 462-469. DOI: 10.1211/jpp.62.04.0008.

2. Sjögren, E.; Abrahamsson, B.; Augustijns, P.; Becker, D.; Bolger, M. B.; Brewster, M,; Brouwers, J.; Flanagan, T.; Harwood, M.; Heinen, C.; Holm, R.; Juretschke, H. P.; Kubbinga, $M$,; Lindahl, A.; Lukacova,V.; Münster, U.; Neuhoff, S.; Nguyen, M. A.; Peer, A. V.; Reppas, C.; Hodjegan, A. R.; Tannergren, C.; Weitschies, W.; Wilson, C.; Zane, P.; Lennernäs, H.; Langguth, P. In vivo methods for drug absorption - comparative physiologies, model selection, correlations with in vitro methods (IVIVC), and applications for formulation/API/excipient characterization including food effects. Eur. J. Pharm. Sci. 2014, 57, 99-151. DOI: 10.1016/j. ejps.2014.02.010.

3. Sugano, K. Theoretical investigation of passive intestinal membrane permeability using Monte Carlo method to generate drug-like molecule population. Int. J. Pharm. 2009, 373, 55-61. DOI: 10.1016/j.ijpharm.2009.02.002.

4. Carlert, S.; Akesson, P.; Jerndal, G.; Lindfors, L.; Lennernäs, H.; Abrahamsson, B. In vivo dog intestinal precipitation of mebendazole: a basic BCS class II drug. Mol. Pharm. 2012, 9, 2903-2011. DOI: 10.1021/mp300224h.

5. Takano, R.; Takata, N.; Saito, R.; Furumoto, K.; Higo, S.; Hayashi, Y.; Machida, M.; Aso, Y.; Yamashita, S. Quantitative analysis of the effect of supersaturation on in vivo drug absorption. Mol. Pharm. 2010, 7, 1431-1440. DOI: 10.1021/mp100109a.

6. Tannergren, C.; Karlsson, E.; Sigfridsson, K.; Lindfors, L.; Ku, A.; Polentarutti, B.; Carlert, S. Biopharmaceutic profiling of salts to improve absorption of poorly soluble basic drugs. J. Pharm. Sci. 2016, 105, 3314-3323. DOI: 10.1016/j.xphs.2016.07.016.

\section{USE OF IN VITRO FLUX MEASUREMENTS TO ASSESS THE COMPLEX INTERPLAY BETWEEN SOLUBILITY, PERMEABILITY AND FORMULATION EFFECTS \\ Konstantin Tsinman}

Pion Inc., Billerica, MA, USA.

The amount and the rate of oral drug absorption is determined by the API flux through the epithelial lining of the small intestine. The flux values depend on the amount of dissolved API that is available at the site of permeation. It also depends on the rate with which the drug penetrates the membranes separating the $\mathrm{Gl}$ tract from the perfusing blood capillaries. The quantity 
of bioavailable API is governed by its dissolution and solubility in the GI fluids when presented at a biorelevant load. Movement of drug from the intestinal lumen into the enterocyte is determined by the drug's effective permeability.

Establishing meaningful correlations between in vivo absorption and in vitro measurements present a significant challenge for pharmaceutical researchers, especially when dealing with poorly soluble compounds. For example, Kataoka et al. showed how biased predictions of absorbed fraction $\left(\mathrm{F}_{\mathrm{a}}\right)$ across a range of danazol formulations occurred when dissolution data alone were considered (1). That study underscores the caution needed when interpreting data generated using USP-type dissolution measurements without considering the associated formulation additives and dissolution/ solubility/permeability values.

The presentation introduced principles and devices (Figure 1) that could be utilized for flux measurements in a systematic and reproducible manner. By utilizing a miniaturized device called micro-FLUX ( $\mu F L U X$, Pion), a "good" IVIVC could be obtained for itraconazole formulations (2-4). For this method, an absorption chamber is introduced that can be used in conjunction with USP I and II dissolution apparatuses to assess drug flux across oral dosage forms (Figure 1). The utility of this device is illustrated by comparing generic telmisartran formulations to the brand name product (5). Flux measurements correctly indicated potential risks for certain generic formulations to meet in vivo BE criteria. Another example of how flux can be used for assessing the risk of drug-drug interactions is when $\mathrm{pH}$ modifying agents are included in the formulation (6).

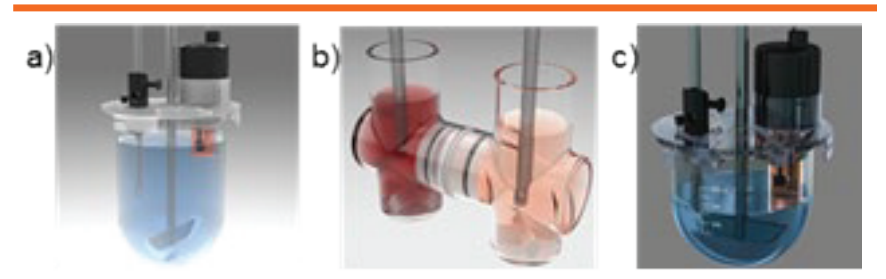

Figure 1. MacroFLUX (a) has absorption chamber (12 mL) inserted in the standard USP 1 or 2 dissolution vessel (800-1000 mL), $\mu F L U X(b)$ uses $16-20 \mathrm{~mL}$ in both side-by-side dissolution and absorption chambers and BioFLUX (c) uses shortened dissolution vessel of USP 1 or $2(200-250 \mathrm{~mL})$. Note: Presented by Tsinman, K.; Borbás, E.; Tsinman, O.; Sinko, B. at the 3rd European Conference on Pharmaceutics, Bologna, Italy, March 25-26, 2019.

Finally, upon exploring a determination of IVIVC based on USP and FDA definitions, it can be shown that the in vitro approach can be used to generate a satisfactory IVIVC. Fa can be estimated from flux measured through artificial membranes that reflect the GI tract (7). Supporting this contention, when using this approach, the predicted Fa of several commercially available formulations of itraconazole and telmisartan were in good agreement with human data.

It was concluded that measuring flux and its dependence on formulations allowed for an assessment of the complex interplay between solubility, permeability and dissolution rate. In particular, flux measurements can provide an early prediction of Fa. It can also be used for formulation ranking, assessing the risk of failing to successfully demonstrate product $\mathrm{BE}$, drug-drug interactions from $\mathrm{pH}$ modifying agents, and the relevance of information derived from other biorelevant in vitro studies.

\section{REFERENCES}

1. Kataoka, M.; Sugano, K.; da Costa Mathews, C.; Wong, J. W.; Jones, K. L.; Masaoka, Y.; Sakuma, S.; Yamashita, S.; Application of dissolution/permeation system for evaluation of formulation effect on oral absorption of poorly water-soluble drugs in drug development. Pharm Res. 2012, 29, 1485-1494. DOI: 10.1007/ s11095-011-0623-2.

2. Tsinman, K.; Tsinman, O.; Lingamaneni, R.; Zhu, S.; Riebesehl, B.; Grandeury, A.; Juhnke, M.; Van Eerdenbrugh, B. Ranking itraconazole formulations based on the flux through artificial lipophilic membrane. Pharm. Res. 2018, 35, 161. DOI: 10.1007/ s11095-018-2440-3.

3. Stewart, A. M.; Grass, M. E.; Mudie, D. M.; Morgen, M. M.; Friesen, D. T.; Vodak, D. T.; Development of a biorelevant, materialsparing membrane flux test for rapid screening of bioavailabilityenhancing drug product formulations. Mol. Pharm. 2017, 14, 2032-2046. DOI: 10.1021/acs.molpharmaceut.7b00121.

4. Stewart, A. M.; Grass, M. E.; Brodeur, T. J.; Goodwin, A. K.; Morgen, M. M.; Friesen, D. T.; Vodak, D. T. Impact of drug-rich colloids of itraconazole and HPMCAS on membrane flux in vitro and oral bioavailability in rats. Mol. Pharm. 2017, 14, 2437-2449. DOI: 10.1021/acs.molpharmaceut.7b00338.

5. Borbás, E.; Nagy, Z. K.; Nagy, B.; Balogh, A.; Farkas, B.; Tsinman, O.; Tsinman, K.; Sinkó, B. The effect of formulation additives on in vitro dissolution-absorption profile and in vivo bioavailability of telmisartan from brand and generic formulations. Eur. J. Pharm. Sci. 2018, 114, 310-317. DOI: 10.1016/j.ejps.2017.12.029.1.

6. Li, J.; Tsinmanm, K.; Tsinman, O.; Wigman, L. Using pH gradient dissolution with in-situ flux measurement to evaluate bioavailability and DDI for formulated poorly soluble drug products. AAPS PharmSciTech. 2018, 19, 2898-2907. DOI: 10.1208/s12249-018-1164-3.

7. Avdeef, A.; Bendels, S.; Di, L.; Faller, B.; Kansy, M.; Sugano, K.; Yamauchi, Y. PAMPA-critical factors for better predictions of absorption. J. Pharm. Sci. 2007, 96, 2893-2909. DOI: 10.1002/ jps.21068. 
USE OF DOGS TO SUPPORT FORMULATION

DEVELOPMENT FOR LARGE MOLECULE ORAL DRUG DELIVERY

\section{Patrick J. Sinko}

Ernest Mario School of Pharmacy, Rutgers University, The State

University of New Jersey, Piscataway, NJ, USA.

Studying the intestinal absorption of large molecule drugs such as oligonucleotides, proteins, peptides, and a variety of nanocarriers is particularly challenging since oral BA can be low and highly variable. However, using an intestinal-vascular access port dog model (IVAP), the systematic investigation of the effects of excipients and other formulation variables on pre-hepatic oral absorption is possible since portal vein sampling is used. With the combination of in vitro methods and ported and regular dogs, three cases were presented to demonstrate the use of the dog model to study large molecule drug absorption. The first case involved excipient screening for an orally administered antisense oligonucleotide (AO). The $A O$ formulation was complex and included multiple performance enhancing (PE) excipients in addition to "inert" excipients (i.e., manufacturing aids, disintegrants, etc.). For this study, the inert excipients remained constant across all studied formulations. Solubilized versions of the formulation were injected into the duodenal port and systemic samples were taken. The BA of the AO increased significantly with the addition of PE excipients. Overall, the BA tripled upon adding the seventh excipient. However, when the eighth PE excipient was added, the BA plummeted to zero. Using fluoroscopy, it was found that the eighth excipient caused massive fluid flux into the intestine resulting in hypermotility pushing the formulation past its absorption window. When an agent was administered to slow motility, the BA was restored.

In the second case study, a large peptide, salmon calcitonin, was studied to determine the target product profile for an oral delivery system $(1,2)$. Preliminary in vitro studies suggested that enzymatic degradation and low permeability were the main obstacles to achieving oral absorption. In the first series of studies, the infusion rate was varied to simulate release rates from very slow to rapid. The data clearly showed that a quick/pulse release profile resulted in significantly higher BA. Regional studies (i.e., injection into the ileum) also clearly showed that BA was highest in the lower small intestine where proteolytic activity was significantly reduced. To reduce proteolytic activity in the upper small intestine, various citric acid formulations were screened to test the hypothesis that reducing intestinal $\mathrm{pH}$ would also reduce the proteolytic activity and improve oral bioavailability. Intestinal pH was monitored using a tethered Heidelberg capsule metering system. The results clearly showed that transiently reducing $\mathrm{pH}$ resulted in higher salmon calcitonin oral bioavailability and that the increased absorption rate directly correlated to the rate and duration of $\mathrm{pH}$ reduction. Thus, the critical product characteristics for an orally administered salmon calcitonin product include rapid release and $\mathrm{pH}$ reduction to $\sim \mathrm{pH} 4.5$, to maximize oral BA.

In the final case study, the oral absorption of nanocarriers, in this case, nanoparticles functionalized with novel peptide ligands, was described (3). The basis for these ongoing studies as well as the preliminary in vitro and in vivo results were presented.

\section{REFERENCES}

1. Lee, Y. H.; Perry, B. A.; Labruno, S.; Lee, H. S.; Stern, W.; Falzone, L. M.; Sinko, P. J. Impact of regional intestinal pH modulation on absorption of peptide drugs: Oral absorption studies of salmon calcitonin in beagle dogs. Pharm. Res. 1999, 16, 1233-1239. DOI: 10.1023/A:1014849630520.

2. Sinko, P. J.; Lee, Y. H.; Makhey, V.; Leesman, G. D.; Sutyak, J. P.; Yu, H.; Perry, B.; Smith, C. L.; Hu, P.; Wagner, E. J.; Falzone, L. M.; McWhorter, L. T.; Gilligan, J. P.; Stern, W. Biopharmaceutical approaches for developing and assessing oral peptide delivery strategies and systems: In vitro permeability and in vivo oral absorption of salmon calcitonin (sCT). Pharm. Res., 1999, 16, 527-533.

3. Sinko, P. J.; Zhang, X.; Szekely, Z. "Intestinal Peptide Targeting Ligands", United States Patent 9,566,349 (February 2017).

USE OF IN SILICO MECHANISTIC MODELS TO SUPPORT INTERSPECIES EXTRAPOLATION OF ORAL BIOAVAILABILITY AND FORMULATION OPTIMIZATION: MODEL EXAMPLE USING

\section{GASTROPLUS}

\section{Viera Lukacova}

Simulation Sciences, Simulations Plus, Inc., Lancaster, CA, USA.

A variety of in vitro, in silico, and animal assays and models are applied within the pharmaceutical drug development process to improve its efficiency from bench to bedside. Mechanistic in silico models provide a unique platform by combining all this information into a single framework for accurate predictions of the complex in vivo drug behavior in animals, healthy subjects, and specific patient populations. Typically, these mechanistic models are used to extrapolate the in vitro and animal in vivo studies to predict the drug behavior in human. The same strategy, however, can be used to extrapolate the drug behavior 
between animal species or even predict the behavior in animals based on in vivo data in humans in cases of extending the use of human medications for veterinary purposes.

The main determinant of successful interspecies extrapolation is a knowledge of the physiological differences between the species in question. Linking the physiology specifications with the physicochemical and biopharmaceutical properties of drugs and drug products enables extrapolating the different in vivo processes influencing drug behavior across species. The majority of prebuilt or commercially available mechanistic models already include built-in physiological information for humans and for several animal species. Some of these parameters come from direct measurements while others are often estimated/deconvoluted from the in vivo behavior of several different compounds.

The presentation highlighted some of the processes affecting the behavior of drugs or drug products in the intestine as implemented in the GastroPlus ${ }^{\circledR}$ software. Information about the intestinal $\mathrm{pH}$ and bile salt concentrations in different species, when linked with the effect of $\mathrm{pH}$ on drug's solubility and the affinity to bile salt micelles, enables the investigator to extrapolate the rate and the extent of intestinal drug dissolution between species. Information about the intestinal $\mathrm{pH}$, absorptive surface areas, sizes and densities of pores helps to translate and explain the interspecies differences in the rates of passive absorption. For the drugs that are substrates for intestinal transporters or are subject to significant intestinal first pass metabolism, the information about the activities of intestinal transporters and enzymes is critical for accurate extrapolation of intestinal absorption and first pass metabolism between the species.

Several examples were presented that showcased the use of GastroPlus for interspecies extrapolation of drug or drug product behavior. Chiang et al. described the use of mechanistic absorption modeling in drug salt form selection and the use of in vivo data in rats to verify these predictions (1). Xia et al. described the use of mechanistic modeling to analyze the in vivo data from dog studies in an effort to evaluate the in vivo behavior of several formulations for the purpose of determining the most likely causes of observed food effect and to help in the selection of a formulation that would minimize this effect (2). Wu et al. described the use of animal data along with a mechanistic model to aid in formulation selection (3). In their study, rat and dog intravenous data were used to validate the prediction of systemic distribution and elimination. The dog data, after oral administration of Dissolution different formulations, was then used to select the most predictive in vitro dissolution experiment to describe the in vivo behavior of the formulation. Finally, Martinez et al. showcased the use of mechanistic absorption models to deconvolute the dissolution and absorption behavior of immediate release formulations of the low solubility drug ciprofloxacin in dog and humans to provide insights into both intersubject variability as well as interspecies differences in ciprofloxacin in vivo behavior $(4,5)$.

\section{REFERENCES}

1. Chiang, P. C.; Wong, H. Incorporation of physiologically based pharmacokinetic modeling in the evaluation of solubility requirements for the salt selection process: a case study using phenytoin. AAPS J. 2013, 15, 1109-1118. DOI: 10.1208/s12248013-9519.

2. Xia, B.; Heimbach, T.; Lin, T. H.; Li, S.; Zhang, H.; Sheng, J.; He, $\mathrm{H}$. Utility of physiologically based modeling and preclinical in vitro/in vivo data to mitigate positive food effect in a BCS class 2 compound. AAPS Pharm. Sci. Tech. 2013, 14, 1255-1266. DOI: 10.1208/s12249-013-0018-2.

3. Wu, C.; Kou, L.; Ma, P.; Gao, L.; Li, B.; Li, R.; Luo, C.; Shentu, J.; $\mathrm{He}, \mathrm{Z}$; S Sun, J. Interspecies prediction of oral pharmacokinetics of different lacidipine formulations from dogs to human: Physiologically based pharmacokinetic modelling combined with biorelevant dissolution. RSC Adv. 2015, 5, 19844-19852. DOI: 10.1039/C4RA16017G.

4. Martinez, M.; Mistry, B.; Lukacova, V.; Polli, J.; Hoag, S.; Dowling, T.; Kona, R.; Fahmy, R. Use of modeling and simulation tools for understanding the impact of formulation on the absorption of a low solubility compound: Ciprofloxacin. AAPS J. 2016, 18, 886897. DOI: 10.1208/s12248-016-9913-2.

5. Martinez, M.; Mistry, B.; Lukacova, V.; Lentz, K. A.; Polli, J.; Hoag, S.; Dowling, T.; Kona, R.; Fahmy, R. Exploring canine-human differences in product performance: Part II: Use of modeling and simulation to explore the impact of formulation on ciprofloxacin in vivo absorption and dissolution in dogs. AAPS J. 2017, 19, 712726. DOI: 10.1208/s12248-017-0055-y.

\section{ASSESSING THE IMPACT OF EXCIPIENTS ON BIOEQUIVALENCE Talia Flanagan}

Previously: Research and Development, AstraZeneca, Macclesfield, Cheshire, UK; Present: UCB Pharma SA, Product Development, Chemin du Foriest, Belgium.

Excipients are selected for inclusion in drug products to perform a variety of functions. However, some excipients can also have an unintended impact on in vivo product performance. Some of these effects would not be detected by standard in vitro dissolution tests, 
necessitating additional consideration when assessing the likelihood of formulation equivalence, especially in the context of biowaivers.

Excipients can potentially impact drug absorption through several mechanisms, including their effect on drug release rate/amount in solution, GI transit time, luminal volumes, effective permeability, and metabolism. There are numerous published reports and review articles that describe such effects (1-6). However, the scientific publications on this topic span a wide range of API properties, formulation types, and excipient quantities. Many of the excipients for which effects on absorption are reported would not normally be used in immediate release solid oral dosage forms. Furthermore, the amounts of excipients shown to impact absorption in some studies are much higher than would normally be used in standard formulations.

Several regulatory guidelines provide criteria for the level of excipient change that can be made without the need for an in vivo BE study. The BCS-based biowaiver guidelines specify limits for permitted excipient changes between test and reference products, within which in vitro dissolution testing is sufficient to demonstrate bioequivalence. While these vary somewhat between regions, for BCS Class 1 drug products, it is usually permitted to make qualitative and quantitative changes to the formulation composition, except for critical excipients that may affect in vivo performance. The guidelines are more restrictive for BCS Class 3 drug products, requiring formulation compositions to be quantitatively and qualitatively similar (7-9). The ICH M9 guideline on BCSBased Biowaivers (currently under development) also provides recommendations about the level of excipient change that can be permitted for BCS Class 1 and 3 drug products (10). The FDA SUPAC guideline (11) specifies maximum acceptable levels of excipient change that are acceptable without an in vivo BE study for all BCS classes (Level 2 changes), provided that certain in vitro dissolution criteria are met.

The criteria established in regulatory guidelines for assessing the allowable excipient differences across formulations are intended to be generally applicable. That is, it can be applied to all immediate release formulations, to all manufacturing processes, and/or to all APIs within a given category. To achieve such a broad set of situations, these criteria need to be based on a more conservative 'worst-case' scenario. Consequently, some changes will not be allowable even if it would not negatively influence product in vivo bioavailability. Applying a mechanistic approach enables the risk of an excipient change affecting absorption to be assessed on a product-specific basis, taking into consideration:

- The mechanism by which the excipient can potentially affect drug absorption;

- The quantity and function of the excipient in the test and reference formulations, compared to the quantity/level at which an effect on absorption has been observed; and

- The absorption properties (site, rate and mechanism) of the API.

Applying a mechanistic and risk-based approach reduces the likelihood of rejecting biowaivers when those formulations that would be equivalent, and it ensures that changes which are likely to impact absorption are more robustly identified. Use of modern biopharmaceutics tools such as in silico PBPK absorption modelling can facilitate a quantitative assessment of excipient impact on product performance in the patient. An example was presented for a BCS Class 4 compound, where PBPK absorption modelling was used to integrate productspecific knowledge with literature data to establish a level of mannitol that could be used in the formulation without significantly impacting in vivo drug absorption.

\section{REFERENCES}

1. Adkin, D. A.; Davis, S. S.; Sparrow, R. A.; Huckle, P. D.; Phillips, A. J.; Wilding, I. R. The effects of pharmaceutical excipients on small intestinal transit. Br. J. Clin. Pharmacol. 1995, 39, 381-387. DOI: 10.1111/j.1365-2125.1995.tb04466.x.

2. García-Arieta, A. Interactions between active pharmaceutical ingredients and excipients affecting bioavailability: Impact on bioequivalence. Eur. J. Pharm. Sci. 2014, 65, 89-97. DOI: 10.1016/j.ejps.2014.09.004.

3. Parr, A.; Hidalgo, I. J.; Bode, C.; Brown, W.; Yazdanian, M.; Gonzalez, M. A.; Sagawa, K.; Miller, K.; Jiang, W.; Stippler, E. $S$. The effects of excipients on the permeability of BCS Class 3 compounds and implications for biowaivers. Pharm Res. 2016, 33, 167-176. DOI: 10.1007/s11095-015-1773-4.

4. Sjögren, E.; Abrahamsson, B.; Augustijns, P.; Becker, D.; Bolger, M. B.; Brewster, M.; Brouwers, J.; Flanagan, T.; Harwood, M.; Heinen, C.; Holm, R.; Juretschke, H. P.; Kubbinga, M.; Lindahl, A.; Lukacova, V.; Münster, U.; Neuhoff, S.; Nguyen, M.A.; Peer, A. V.; Reppas, C.; Hodjegan, A. R.; Tannergren, C.; Weitschies, W.; Wilson, C.; Zane, P.; Lennernäs, H.; Langguth, P. In vivo methods for drug absorption - Comparative physiologies, model selection, correlations with in vitro methods (IVIVC), and applications for formulation/API/excipient characterization including food effects. Eur. J. Pharm. Sci. 2014, 57, 99-151. DOI: 10.1016/j. ejps.2014.02.010. 
5. Vaithianathan, S.; Haidar, S. H.; Zhang, X, Jiang, W.; Avon, C.; Dowling, T.C, Shao, C.; Kane, M.; Hoag, S. W.; Flasar, M. H.; Ting, T. Y.; Polli, J. E. Effect of common excipients on the oral drug absorption of biopharmaceutics classification system class 3 drugs cimetidine and acyclovir. J. Pharm. Sci. 2016, 105, 9961005. DOI: 10.1002/jps.24643.

6. Zhang, W.; Li, Y.; Zou, P.; Wu, M.; Zhang, Z.; Zhang, T. The effects of pharmaceutical excipients on gastrointestinal tract metabolic enzymes and transporters-an update. AAPS J. 2016, 18, 830843. DOI: 10.1208/s12248-016-9928-8.

7. Guideline on the investigation of bioequivalence (CPMP/QWP) EWP/1401/98 Rev. 1). European Medicines Agency, London UK; 2010.

8. Waiver of In Vivo Bioavailability and Bioequivalence Studies for Immediate-Release Solid Oral Dosage Forms Based on a Biopharmaceutics Classification System. Guidance for Industry: U.S. Department of Health and Human Services, Food and Drug Administration, Center for Drug Evaluation and Research (CDER), Silver Spring MD, 2017.

9. Immediate Release Solid Oral Dosage Forms - Scale-Up and Postapproval Changes: Chemistry, Manufacturing, and Controls, In Vitro Dissolution Testing, and In Vivo Bioequivalence Documentation. Guidance for Industry: U.S. Department of Health and Human Services, Food and Drug Administration, Center for Drug Evaluation and Research (CDER), Silver Spring MD, 1995.

10. Biopharmaceutics Classification System Based Biowaiver. Health Canada Guidance Document: 2014.

11. ICH Final endorsed Concept Paper M9: Biopharmaceutics Classification System-based Biowaivers, 7 October 2016.http:// www.ich.org/fileadmin/Public_Web_Site/ICH_Products/ Guidelines/Multidisciplinary/M9/ICH_M9_Concept_paperfinal_70ct2016.pdf

\section{ASSESSING PRODUCT PERFORMANCE OF NON- SYSTEMICALLY ACTING DRUGS David Sperry}

Small Molecule Design \& Development, Eli Lilly and Company,

Indianapolis, IN, USA.

As introduced by Charmot, non-systemically acting drugs can be conveniently classified into five groups: sequestering agents, ligands of intestinal enzymes, enzymes, minimally absorbed and rapidly metabolized drugs, and apical targets (1). As the names imply, drugs in each of these categories achieve their therapeutic effect through different mechanisms of action.

A property that nearly all these drugs share is that they are not constrained to the epithelial permeability requirements influencing the effectiveness of most oral therapeutic agents. Removing this obstacle allows for a much broader molecular space. Proteins, peptides and functionalized polymers have all been used as nonsystemic agents.

A complication of the fact that these drugs are not absorbed is that plasma concentrations cannot be used as a measure of in vivo product performance. This is particularly problematic when assessing the impact of changes in the drug product. While PK studies are often utilized as the "gold standard" for equivalence of oral therapeutic agents, developers of non-systemic agents must rely on other measures to demonstrate equivalence. A framework for dealing with these issues was introduced and is shown in Figure 1. A given dosage form design will have a particular drug release mechanism. After drug is released, it must arrive at the site of action where it can have the intended pharmacological action. Understanding these steps mechanistically provides a basis to determine how physical properties, manufacturing process and product performance measures can be assembled to create an appropriate control strategy.

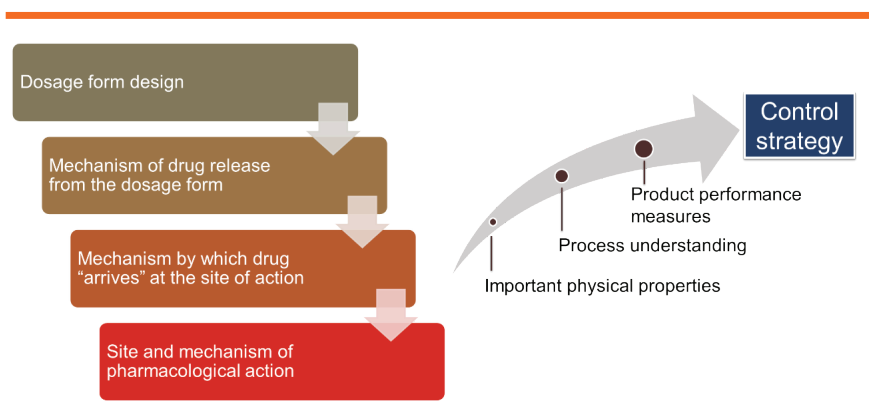

Figure 1. Recommended framework to assess and control product performance for non-systemically acting drugs.

A few examples were discussed including: orlistat (2), ATI7505 (3), ezetimibe (4), Keyexalate (polystyrene sulfonate) (5), and liprotamase (6). In each case, the design of the dosage form, the release mechanism and the arrival at the site of action was considered. The control strategy for each one was then discussed in the context of what is known about the release mechanism. Liprotamase, which contains a novel cross-linked enzyme, was discussed in some detail. This case illustrated how the degree of crosslinking is a critical parameter that may impact in vivo drug release.

In summary, while non-systemically acting drugs present unique challenges for product performance assessment, an approach that considers the relevant mechanism was shown to be highly valuable. 


\section{REFERENCES}

1. Charmot, D. Non-systemic drugs: a critical review. Curr. Pharm. Des.2012,18, 1434-1445. DOI:10.2174/138161212799504858.

2. XENICAL Package Insert, Roche Pharmaceuticals, Revised January 2009.

3. Camilleri, M.; Vazquez-Roque, M. I.; Burton, D.; Ford, T.; McKinzie, S.; Zinsmeister, A. R.; Druzgala, P. Pharmacodynamic effects of a novel prokinetic 5-HT receptor agonist, ATI-7505, in humans. Neurogastroenterol. Motil. 2007, 19, 30-38. DOI: 10.1111/j.1365-2982.2006.00865.x.

4. Zetia Package Insert, Merck \& Co, Inc., Revised August 2013.

5. Keyexalate Package Insert, Sanofi-Aventis, Revised 2009.

6. Hetrick, E. M.; Sperry, D. C.; Nguyen, H. K.; Strege, M. A. Characterization of a novel cross-linked lipase: impact of crosslinking on solubility and release from drug product. Mol. Pharm. 2014, 11, 1189-1200. DOI: 10.1021/mp4006529.

\section{IN SILICO BE: STRENGTHS, WEAKNESSES, POTENTIAL APPLICATIONS Masoud Jamei}

Certara UK Limited (Simcyp Division), Sheffield, UK.

BE studies are typically conducted to assess whether two formulations containing the same dose of same chemical entity, generally in the same dosage form, are interchangeable as defined by their respective rates and extent of absorption. Typically, these studies are conducted in healthy volunteers and can occur across all stages of drug (product) development. For example, they can be used to link early and late phase clinical formulations, compare clinical versus to be marketed formulations, evaluate changes in product formulation (tablet vs. capsule, qualitative and quantitative modifications in product composition), differences in manufacturing sites, and to compare generic versus branded drug products. The number of BE studies conducted can be high. However, modelling and simulation (in silico or virtual) can significantly help to reduce, refine and replace these in vivo studies.

Virtual $B E$ can be performed using mechanistic PBPK models. These models can integrate a range of physiological, biological and genetic data of the target population with that of the drug API/formulation (1). The recent success of PBPK models is mainly due to linking those models to IVIVE techniques. IVIVE allows the investigator to integrate drug-specific parameters (such as its physicochemical properties as well as its PK characteristics that influence its absorption, metabolism, transport, etc.) and its formulation-specific attributes with the system (population) characteristics and trial design to predict in vivo dissolution and systemic drug exposure (2). In the same line, physiologically-based IVIVC approaches can address many issues of traditional IVIVC approaches by disentangling dissolution, absorption and metabolism processes (3).

In vitro dissolution data can directly be incorporated into PBPK models. Nevertheless, since the dissolution data are mapped into PBPK models, it will not be possible to consider known physiological and anatomical variability in the $\mathrm{Gl}$ tract and their potential impacts on in vivo dissolution. As a result, the scope of virtual BE studies will be limited. On the other hand, the biopharmaceutical IVIVE approach is in a better position to inform PBPK models and enable virtual BE simulations. Biopharmaceutical IVIVE applications have already been reported, modelling various solubility and dissolution experiments for posaconazole, ketoconazole and dipyridamole $(4,5)$.

Virtual BE studies require the use of mechanistic absorption models and a knowledge of the anatomy, biology and physiology of the GI tract. Also required is an appreciation of the variability associated with these parameters across the population(s) of interest. Moreover, to account for inter-occasion variability, the knowledge of biological and physiological parameters changes within subjects are required. While there remain many gaps in our knowledge in these areas, it is still possible to use virtual BE simulations to compare formulations performance and to assess the BE for some formulations.

Doki and colleagues have used virtual BE to examine achlorhydria-related BE disparity across formulations of levothyroxine and nifedipine. They evaluated virtual product BE in healthy volunteers and in Japanese elderly that presented with a high incidence of achlorhydria. While the nifedipine control-released reference and test formulations were bioequivalent in healthy volunteers, these same products were inequivalent in the Japanese elderly population (6).

Virtual BE studies can also be applied to other routes of administrations such as dermal, inhalation and ocular dosing. Such trials can help avoid the need to conduct unnecessary studies, potentially expand biowaivers, consider pharmacodynamics as an endpoint, and to provide insights into drug products performance in various healthy and patient populations. 


\section{REFERENCES}

1. Jamei, M.; Dickinson, G. L.; Rostami-Hodjegan, A. A framework for assessing interindividual variability in pharmacokinetics using virtual human populations and integrating general knowledge of physical chemistry, biology, anatomy, physiology and genetics: A tale of 'bottom-up' vs 'top-down' recognition of covariates. Drug Metab. Pharmacokinet. 2009, 24, 53-75. DOI: 10.2133/ dmpk.24.53.

2. Rostami-Hodjegan, A. physiologically based pharmacokinetics joined with in vitro-in vivo extrapolation of ADME: A marriage under the arch of systems pharmacology. Clin. Pharmacol. Ther. 2012, 92, 50-61. DOI: 10.1038/clpt.2012.65.

3. Mistry, B.; Patel, N.; Jamei, M.; Rostami-Hodjegan, A.; Martinez, M. N. Examining the use of a mechanistic model to generate an in vivo/in vitro correlation: journey through a thought process. AAPS J. 2016, 18, 1144-1158. DOI: 10.1208/s12248-016-9930-1.

4. Hens, B.; Pathak, S. M.; Mitra, A.; Patel, N.; Liu, B.; Patel, S.; Jamei, M.; Brouwers, J.; Augustijns, P.; Turner, D. B. In silico modeling approach for the evaluation of gastrointestinal dissolution, supersaturation, and precipitation of posaconazole. Mol. Pharm. 2017, 14, 4321-43333. DOI: 10.1021/acs. molpharmaceut.7b00396.

5. Pathak, S. M.; Schaefer, K. J.; Jamei, M.; Turner, D. B. Biopharmaceutic IVIVE - mechanistic modelling of singleand two-phase in vitro experiments to obtain drug-specific parameters for incorporation into PBPK models. J. Pharm. Sci. 2018, 108, 1604-1618. DOI: 10.1016/j.xphs.2018.11.034.

6. Doki, K.; Darwich, A. S, Patel, N.; Rostami-Hodjegan, A. Virtual bioequivalence for achlorhydric subjects: The use of PBPK modelling to assess the formulation-dependent effect of achlorhydria. Eur. J. Pharm. Sci. 2017, 109, 111-120. DOI: 1016/j. ejps.2017.07.035.

\section{ABSORPTION MODELLING FOR VIRTUAL TRIALS. CURRENT APPLICATIONS AND A VISION FOR THE FUTURE \\ Xavier Pepin}

AstraZeneca, Macclesfield, UK.

PBPK modelling in the field of drug substance and drug product quality is an important tool which can be used to define acceptable specifications in terms of critical material attributes (CMA) or critical process parameters (CPP). The model development is supported by in vivo evaluation of clinical batch variants outside of the normal operating range (the knowledge space). If the PBPK model can successfully predict the drug product clinical performance within this knowledge space, based on inputs related to the product CMA or CPP, it can then be used to define the size of the safe space, i.e., the space within which all the product batches are considered bioequivalent to the clinical reference (Figure 1).

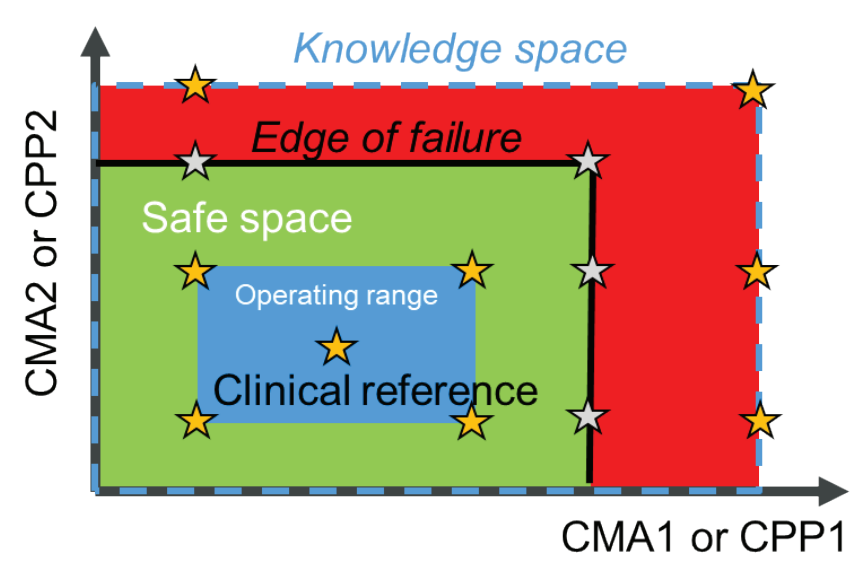

Figure 1. Knowledge space, safe space, operating range and edge of failure versus CMA and CPP. Orange stars shows in vivo evaluation and grey stars are virtual batches.

The only way to know the size of the safe space (or position the edge of failure) is to manufacture variants that are sufficiently different from the clinical reference so that they are not bioequivalent to this reference. In this case, there is an opportunity to link the CMA or CPP to in vivo drug exposure and therefore establish an IVIVC.

Alternatively, when all tested batch variants are bioequivalent to the clinical reference, the safe space is at least as large as the space defined by the batches tested. However, the absolute size of the safe space remains unknown. In this situation, no IVIVC can be established since, by definition, the in vitro variations observed do not translate into in vivo meaningful differences (1). However, the observed "safe space" can provide the flexibility needed to permit drug product manufacture with consistent quality for the patients during the life cycle of the product. The regulatory flexibility within the product safe space can be used to make changes to product specifications, compositions, or processes. Specifically, as long as the proposed changes are contained within the safe space, BE evaluations can be waived (2).

In PBPK models, parameters can be classified as: a) drug and drug substance related, b) drug product related and c) system parameters (related to the modelled physiology and population). Some drug parameters are not independent from the system parameters, such as the blood to plasma partitioning, or the fraction unbound in plasma, which could depend on the species, age or the disease state. The dissolution rate of drug products is also affected by the systems parameters such as $\mathrm{pH}$, volume, transit or bile salt concentration. 
A mechanistic model is one which, based on physicochemical first principles, allows an investigator to dynamically calculate a phase transition or a chemical reaction that depends on the systems parameters. For instance, the use of a Weibull function to fit observed in vitro dissolution versus time profile is not a mechanistic approach. With such a model, the predicted in vivo dissolution profile will only depend on the time spent in the system. In contrast, models such as Takano's Z-factor (3) or a Product Particle Size Distribution (P-PSD) approach are considered mechanistic since the rate and extent of predicted in vivo dissolution will depend on the drug dose, luminal volume, GI transit, intestinal permeability, and the other local conditions governing drug solubility or precipitation rate (4).

The development of PBPK models to support specification setting is proposed to be based on pilot clinical studies where high-quality data are generated in a small number of subjects. The use of relevant biomarkers is also encouraged since it supports a more mechanistic interpretation of observed PK profiles. Two examples were developed in the presentation. The first example illustrated the use of a SmartPill ${ }^{\circledR}$ as a biomarker for stomach $\mathrm{pH}$, which allowed to understand the role of gastric $\mathrm{pH}$ in modulating stomach dissolution, leading to observed variations in dog and human systemic exposure to acalabrutinib. The smartpill temperature reading was also used to calculate the time and volume of water consumed by healthy volunteers. This information was used to explain lag times in the gastric emptying of the drug.

The second example was the use of PBPK modelling to generate dissolution specifications for Zurampic ${ }^{\circledR}$. The dissolution rate of various batches was integrated in the PBPK model using a P-PSD approach, which proved superior to either Weibull functions or Z-factors for predicting in vivo behavior. Virtual BE trials were optimized to integrate within subject variability in terms of stomach $\mathrm{pH}$ and transit time, both which were absent from the default PBPK model setting.

The conclusion of this presentation summarized the findings and opened new paths for improved mechanistic PBPK models (Figure 2). With the use of biomarkers, the variability in exposure can be explained and integrated in the model. This can divert assessments from a statistical data treatment to one that relies upon a mechanistic understanding. Such an approach creates several important new opportunities: 1) to conduct more appropriate virtual BE testing, i.e., expanding on the pilot study to cover more relevant clinical scenarios and thereby avoid unnecessary human testing, and 2) use PBPK approaches to support personalized medicine using models adapted to an individual rather than to a population.
Mechanistic integration of dissolution (P-PSD, Z-factor...)

The right biomarker for the right drug : pH,

transit, bile salt, enzyme expression etc

Use Individual disposition parameters
Move from mechanistic

understanding of populations

to personalized medicine

Run a pilot clinical study with different drug

products. Build a mechanistic absorption model

to explain observed exposure

Run virtual cross-over BE trials to define edge of

failure of CMA and CPP on larger populations with

right variability of key parameters

Figure 2. A vision for the future of PBPK modelling. 
While modelling approaches are applicable to all administration routes, mechanistic models supporting non-oral administration need further development and use within a regulatory environment.

\section{REFERENCES}

1. Dickinson, P. A.; Lee, W. W.; Stott, P. W.; Townsend, A. I.; Smart, J. P.; Ghahramani. P.; Hammett, T.; Billett, L.; Behn, S.; Gibb, R. C.; Abrahamsson, B. Clinical relevance of dissolution testing in quality by design. AAPS J. 2008, 10, 380-390. DOI: 10.1208/ s12248-008-9034-7.

2. Pepin, X. J.; Flanagan, T. R.; Holt, D. J.; Eidelman, A.; Treacy, D.; Rowlings C. E. Justification of drug product dissolution rate and drug substance particle size specifications based on absorption PBPK modeling for lesinurad immediate release tablets. Mol. Pharm. 2016, 13, 3256-3269. DOI: 10.1021/acs. molpharmaceut.6b00497.

3. Takano, M.; Yumoto, R.; Murakami, T. Expression and function of efflux drug transporters in the intestine. Pharmacol. Ther. 2006, 109, 137-161. DOI: 10.1016/j.pharmthera.2005.06.005.

4. Stillhart, C.; Pepin, X.; Tistaert, C.; Good, D.; Van Den Bergh, A.; Parrott, N.; Kesisoglou, F. PBPK absorption modeling: establishing the in vitro-in vivo link-industry perspective. AAPS J. 2019, 21, 19. DOI: 10.1208/s12248-019-0292-3.

\section{PRODUCT UNDERSTANDING AS A MECHANISM FOR DEVELOPING DISSOLUTION TEST METHOD Raafat Fahmy}

United States Food and Drug Administration, Center for Veterinary Medicine, Rockville, MD, USA.

Optimization of product formulation reflects the combination of product design strategies and the information provided through use of in vitro dissolution testing procedures. When developing a dissolution method, the relationship between in vitro product performance, drug physicochemical properties, product formulation, product in vivo dissolution behavior, and the biological variables influencing oral drug absorption needs to be understood. In turn, the nature of these relationships is defined by the thermodynamic (equilibrium) API solubility, the ability of the drug to move across a biological membrane (enterocyte or the liver), and the fraction of the dose that successfully moves into the systemic circulation.

Ideally, the dissolution method should be established with the goal of being discriminating (identifying changes in product CQAs), biopredictive (predictive of those changes that will influence in vivo product performance), and sensitive to changes in product integrity during its shelf life (1). This translates to the need for thinking critically about the drug substance, excipients, and the manufacturing process (including the high or low risk variables as it relates to product CQAs). Setting specifications should be aligned with the dosage form's performance objective(s), ensure batch-to batch consistency and signal potential problems that can alter in vivo bioavailability (2).

Test conditions need to be designed from the perspective of drug substance attributes such as solubility, stability in the medium, etc. Regarding formulations, it is important to consider how the dosage form will behave within the dissolution vessels. The in vitro dissolution profile may reflect the product physical and mechanical properties such as surface area, density, particle size, the chemical properties (such as the interaction between the API and the excipients in the dissolution apparatus), the purity, grade, and stability of the excipients, and the release mechanism being incorporated into the product being tested.

When developing a dissolution method, it is important to consider the medium, the apparatus, and the test conditions such as agitation speed, temperature, and medium sampling method. Regarding the apparatus used and the agitation speed, one needs to factor the hydrodynamics of the system. This includes confirming that there is no problem with floating particles, coning, product sticking to the vessels' interior, bubbles, foaming, or crosslinking. Validation procedures that need to be followed and the apparatus-specific factors to be controlled are described in the USP <711> and <1092>. In terms of the medium, it should ideally be biorelevant, compatible with the dosage form, contain a buffer system that can maintain a constant $\mathrm{pH}$ throughout the duration of the study, have an ionic strength that does not interfere with the dissolution of the dosage form, and if solubilizers or co-solvents are needed (e.g., SLS, polysorbate, C-Tab, solutol) should be used in an amount that does not camouflage potential changes in product CQAs.

Risk assessment models are used to identify potential variables that may impact the performance and discriminating capability of the method. An Ishikawa diagram (3) lists all the variables pertaining to the API and drug product characteristics, categorizing the potential cause of any potential problems. A Failure Mode and Effect Analysis (FMEA) model is used to identify the cause of failure and rank the risk that may lead to poor product performance. It can also be used to identify potential aspects of the in vitro dissolution test method that are 
most critical in terms of defining the in vitro release profile. Examples of parameters that may be highly influential include medium composition, $\mathrm{pH}$, surfactant concentration, and RPM (3). The effect of critical method parameters on method robustness and discriminating capability can be verified through design of experiment studies using high risk factors as independent variables. It is these factors that need to be incorporated into the method control strategy to ensure reliability and repeatability of the results.

\section{REFERENCES}

1. USP Chapter <1092> The Dissolution Procedure: Development and Validation. In The United States Pharmacopeia and National Formulary USP 41-NF 36; The United States Pharmacopeial Convention, Inc.: Rockville, MD, 2018; pp 7178-7198.

2. Dissolution Testing of Immediate Release Dosage Forms. Guidance for Industry; U.S. Department of Health and Human Services, Food and Drug Administration, Center for Drug Evaluation and Research (CDER), U.S. Government Printing Office: Washington, DC, 1997.

3. Fahmy, R.; Martinez, M. N. Primer on the science of in vitro dissolution testing of oral dosage forms and factors influencing its biological. Dissolution Technol. 2019, 26, 14-26. DOI: 10.14227/DT260119P14.

\section{USING IN VITRO TOOLS TO PREDICT PULMONARY DRUG DELIVERY Ben Forbes}

Institute of Pharmaceutical Science, King's College London, London, UK.

\section{Pulmonary drug delivery}

Inhalation is the route of administration predominately used when targeting drugs to the lungs for the treatment of respiratory diseases such as asthma, chronic obstructive pulmonary disease, cystic fibrosis and infection. A wide variety of inhaler devices and aerosol formulation types (powders, solutions, suspensions) are licensed as medicinal products (1). Inhaled medicines differ from oral dosage forms in that they are generally more complex, expensive and less intuitive for patients to use. In addition, not all the nominal dose is delivered to the site of action (i.e. some of the aerosolized drug either fails to reach the lungs or is exhaled), and both the delivered dose and its pulmonary distribution can vary widely between users. For drug deposited in the airways, non-absorptive clearance (principally mucociliary clearance) can remove a significant proportion of the dose, much of which is subsequently swallowed (which can lead to systemic drug exposure). In the alveolar region, aerosol particles deposit on a thin fluid film that undergoes expansion and contraction cycles.

A benefit (but also a complication) of respiratory medicines is that the site of delivery is also the location of disease, which can significantly influence drug deposition and disposition. Another challenge is that measurement of local bioavailability is technically difficult, and systemic concentrations fail to reflect target site delivery since it is 'downstream' from the site of action (2).

\section{Aerosol properties of inhaled medicines}

In vitro characterization of inhaled products relies predominately on particle sizing techniques that provide emitted dose information on such parameters as fine particle fraction, mass median aerodynamic diameter and aerodynamic particle size distribution (3). A variety of in vitro methods are available for evaluating pharmaceutical aerosols including different impactors and the reliability of these measurements has been investigated (4). The emphasis on physical aerosol properties reflects the importance of particle size in determining lung dose and regional deposition, thereby allowing for these parameters to be used to evaluate the bioequivalence of inhaled medicines. Although principally considered as QC tools, there has been interest in determining whether an IVIVC can be established (5), especially when utilized with mouth-throat models (6). There is also emerging interest in methods of structural analysis for respirable powders and aerosolization dynamics/particle chemistry for aerosols generated from liquids (7).

\section{In vitro tools for inhalation biopharmaceutics}

Key questions for the biopharmaceutics of inhaled drug delivery were articulated by Lewis Schanker forty years ago (8), including: 1) interaction of a droplet on deposition; 2) rate of particle dissolution; 3) influence of lung lining fluid; 4) nature of pores in different regions of the lung; 5) effect of disease; 6) type and distribution of transporters; and 7) effect of inhaled medicines on lung permeability. Progress in understanding these influences has been well reviewed (9-11). A variety of in vitro, ex vivo and in vivo models are available for studying the interaction of drugs with the lungs (12), with the pay-off being between complexity, cost and relevance (13). Models (and their applications) include: non-cellular fluids (solubility, dissolution); homogenates (binding, metabolism); cell models (permeability, metabolism, toxicity); tissue models (mucociliary clearance, permeability); lung slices (drug uptake, metabolism); and isolated lungs (absorptive transfer, metabolism). Although tissue binding and 
non-absorptive clearance are important determinants of product BA, techniques for characterizing inhaled medicines in terms of drug dissolution and permeability have attracted a great deal of attention.

\section{Measuring dissolution of aerosol medicines}

A variety of dissolution methods are available, differing in terms of their mode of aerosol collection, dissolution and data analysis. It is recognized that doses should be well dispersed and aerosol fractions defined (14). Early considerations regarding dissolution assays for orally inhaled drug products $(15,16)$ have matured in recent years with an explosion of interest $(14,17)$. The importance of dissolution is now being explored as a QC parameter, for establishing an IVIVC, and as an input parameter for mechanistic modelling. Recent developments include the design of apparatuses for collecting aerosols and for performing dissolution studies (18), and the design of biorelevant dissolution media $(17,19,20)$. Future needs include method standardization and the establishment of criteria for evaluating the biopharmaceutical and clinical relevance of the resulting information.

\section{Measuring the permeability of inhaled drug compounds}

Drug permeability or absorptive transport in the lungs is generally measured using cell lines or evaluated ex vivo using isolated perfused lungs (12). The most popular respiratory cell lines were established as models over a decade ago (21). However, an advantage for using organ-specific cell lines has not been demonstrated either in studies comparing permeability results across different cell lines versus that in isolated lungs (22) or as compared to that of an in vivo absorption model (23). Nevertheless, a variety of new cell culture-based models are currently being evaluated, often in combination with aerosol delivery to air-interfaced cells. Questions remain regarding differences in regional permeability and how to evaluate such differences using in vitro systems, the impact of permeability on bioavailability performance (11), and whether transporters are influential in the lung absorption of some inhaled compounds (24).

\section{CONCLUSION}

In vitro methods to predict pulmonary absorption are considerably less developed than that of the oral absorption biopharmaceutics toolkit. Two drivers for change are providing impetus to develop methods for studying inhalation biopharmaceutics: 1) consideration of a putative inhalation biopharmaceutics classification system (10); and 2) the emergence of simulation science and the requirement for obtaining the input necessary to support the use of mechanistic models to predict inhaled drug delivery performance (11).

\section{REFERENCES}

1. Lexmond. A.; Forbes, B. Drug delivery devices for inhaled medicines. In Pharmacology and Therapeutics of Asthma and COPD, Handbook of Experimental Pharmacology; C.P. Page, C.P.; Barnes, P.J.; Eds. New York: Springer, Vol 237, 2016; pp 266-280.

2. Forbes, B.; Asgharian, B.; Dailey, L. A.; Ferguson, D.; Gerde, P.; Gumbleton, M.; Hardy, C.; Hassall, D.; Gustavsson, L.; Jones, R.; Lock, R.; Maas, J.; McGoverin, T.; Pitcairn, G.; Somers, G.; Wolff, $R$. Challenges in inhaled product development and opportunities for open innovation, Adv. Drug Deliv. Rev. 2011 63, 69-87. DOI: 10.1016/j.addr.2010.11.004.

3. Forbes, B.; Backman, P.; Christopher, D.; Dolovich, M.; Bing, L.; Morgan, B. In vitro testing for orally inhaled products: developments in science-based regulatory approaches. AAPS J. 2015, 17, 837-852. DOI: 10.1208/s12248-015-9763-3.

4. Nichols, S. C.; Mitchell, J. P.; Sandell, D.; Andersson, P. U.; Fischer, M.; Howald, M.; Pengilley, R.; Krüger, P. A multi-laboratory in vitro study to compare data from abbreviated and pharmacopeial impactor measurements for orally inhaled products: a report of the European Aerosol Group (EPAG). AAPS Pharm. Sci. Tech. 2016, 17, 1383-1392. DOI: 10.1208/s12249-015-0476-9.

5. Olsson, B.; Borgstrom, L.; Lundback, H.; Svensson, M. Validation of a general in vitro approach for prediction of total lung deposition in healthy adults for pharmaceutical inhalation products. J. Aerosol. Med. Pulmon. Drug Deliv. 2013, 26, 355369. DOI: 10.1089/jamp.2012.0986.

6. Wei, X,; Hindle, M.; Kaviratna, A.; Huynh, B. K., Delvadia, R. R.; Sandell, D.; Byron, P. R.. In vitro tests for aerosol deposition. VI: realistic testing with different mouth-throat models and in vitro in vivo correlations for a dry powder inhaler, metered dose inhaler, and soft mist inhaler. J. Aerosol. Med. Pulmon. Drug Deliv. 2018, 31, 358-371. DOI: 10.1089/jamp.2018.1454.

7. Buttini, F.; Miozzi, M.; Balducci, A. C.; Royall, P. G.; Colombo, P.; Brambilla, G.; Bettini, R.; Forbes, B. Differences in physical chemistry and dissolution rate of solid particle aerosols from solution inhalers. Int. J. Pharm. 2014, 465, 42-51. DOI: 10.1016/j. ijpharm.2014.01.033.

8. Schanker, L. S. Drug absorption from the lung. Biochem. Pharmacol. 1978, 27, 381-385.

9. Patton, J. S.; Brain, J. D.; Davies, L. A.; Fiegel, J.; Gumbleton, M. Kim, K.-J.; Sakagami, M.; Vanbever, R.; Ehrhardt, C. The particle has landed-characterizing the fate of inhaled pharmaceuticals. J. Aerosol. Med. Pulmon. Drug Deliv. 2010, 23, S71-S87. DOI: 10.1089/jamp.2010.0836.

10. Hastedt, J.E.; Bäckman. P.; Clark. A.R.; Doub, W.; Hickey, A.; Hochhaus, G.; Kuehl, P. J.; Lehr, C-M.; Mauser, P. McConville, J.; Niven, R.; Sakagimi, M.; Weers, J. G. Scope and relevance of a pulmonary biopharmaceutical classification system AAPS/FDA/ 
USP Workshop March 16-17th. AAPS Open 2016, 2, 1-20. DOI: 10.1186/s41120-015-0002-x.

11. Bäckman, P.; Couet, W.; Forbes, B.; de Kruijf,W.; Arora, S.; Paudel, A. Advances in experimental and mechanistic computational models to understand pulmonary exposure to inhaled drugs. Eur. J. Pharm. Sci. 2018, 113, 41-52. DOI: 10.1016/j.ejps.2017.10.030.

12. Sakagami, M. In vivo, in vitro and ex vivo models to assess pulmonary absorption and disposition of inhaled therapeutics for systemic delivery. Adv. Drug Deliv. Rev. 2006, 58, 1030-1060. DOI: 10.1016/j.addr.2006.07.012.

13. Tronde, A.; Bosquillon, C.; Forbes, B. The isolated perfused lung for drug absorption studies. In Drug Absorption Studies - In Situ, In Vitro and In Silico Models; Ehrhardt, C.; Kim, K.J.; Eds. New York: Springer, 2008; pp 135-163.

14. Forbes, B.; Hauet-Richer, N.; Buttini, F. Dissolution: a critical performance characteristic of inhaled products? In Pulmonary Drug Delivery: Advances and Challenges, Nokhodchi, A.; Martin, G.P.; Eds. West Sussex, United Kingdom; John Wiley \& Sons, Ltd, 2015; pp 223-240.

15. Son, Y. J.; McConville, J. T. Development of a standardized dissolution test method for inhaled pharmaceutical formulations. Int. J. Pharm. 2009, 382, 15-22. DOI: 10.1016/ j.ijpharm.2009.07.034.

16. Riley, T.; Christopher, D.; Arp, J.; Casazza, A.; Colombani, A.; Cooper, A.; Dey, M.; Maas, J.; Mitchell, J.; Reiners, M.; Sigari, N.; Tougas, T.; Lyapustina, S. Challenges with developing in vitro dissolution tests for orally inhaled products (OIPs). AAPS Pharm. Sci. Tech. 2012, 13, 978-989. DOI: 10.1208/s12249-012-9822-3.

17. Radivojev, S.; Zellnitz, S.; Paudel, A.; Fröhlich, E.. Searching for physiologically relevant in vitro dissolution techniques for orally inhaled drugs. Int. J. Pharm. 2019, 556, 45-56. DOI: 10.1016/ j.ijpharm.2018.11.072.

18. Velaga, S.P.; Djuris, J.; Cvijic, S.; Rozou, S.; Russo, P.; Colombo, G.; Rossi, A. Dry powder inhalers: An overview of the in vitro dissolution methodologies and their correlation with the biopharmaceutical aspects of the drug products. Eur. J. Pharm. Sci. 2018, 113, 18-28. DOI: 10.1016/j.ejps.2017.09.002.

19. Hassoun, M.; Royall, P. G.; Parry, M.; Harvey, R. D.; Forbes, B. Design and development of a biorelevant simulated human lung fluid. J. Drug Deliv. Sci. Technol. 2018, 47, 485-491. DOI: 10.1016/j.jddst.2018.08.006.

20. Kumar, A.; Terakosolphan, W.; Hassoun, M.; Vanderam K. K.; Novicky, A,; Harvey, R.; Royall, P. G.; Bicer, E. M.; Eriksson, J.; Edwards, K.; Valkenborg, D.; Nelissen, I.; Hassall, D.; Mudway, I. S.; Forbes, B. A. Biocompatible synthetic lung fluid based on human respiratory tract lining fluid composition. Pharm. Res. 2017, 34, 2454-2465. DOI: 10.1007/s11095-017-2169-4.

21. Forbes, B.; Ehrhardt, C. Human respiratory epithelial cell culture for drug delivery pplications. Eur. J. Pharm. Biopharm. 2005, 60, 193-205. DOI: 10.1016/j.ejpb.2005.02.010.

22. Manford, F.; Tronde, A.; Jeppsson, A. B.; Patel, N.; Johansson,
F.; Forbes, B. Drug permeability in 16HBE14o- airway cell layers correlates with absorption from the rat lung. Eur. J. Pharm. Sci. 2005, 26, 215-220. DOI: 10.1016/j.ejps.2005.07.010.

23. Tronde, A.; Nordén, B.; Marchner, H.; Wendel, A. K.; Lennernäs, H.; Bengtsson, U. H. Pulmonary absorption rate and bioavailability of drugs in vivo in rats: structure-absorption relationships and physicochemical profiling of inhaled drugs, J. Pharm. Sci. 2003, 92, 1216-1233. DOI: 10.1002/jps.10386.

24. Ehrhardt, C.; Bäckman, P.; Couet, W.; Edwards, C.; Forbes, B.; Fridén, M.; Gumbleton, M.; Hosoya, K.; Kato, Y.; Nakanishi, T.; Takano, M.; Terasaki, T.; Yumoto, R. Current progress toward a better understanding of drug disposition within the lungs: summary proceedings of the 1st Workshop on Drug Transporters in the Lungs. J. Pharm. Sci. 2017, 106, 2243-2244. DOI: 10.1016/j.xphs.2017.04.011.

\section{TRANSLATIONAL APPLICATIONS OF TISSUE CHIP TECHNOLOGIES Murat Cirit}

Massachusetts Institute of Technology, Cambridge, MA, USA.

The Translational Center of Tissue Chip Technologies $\left(\mathrm{TC}^{2} \mathrm{~T}\right)$ is spearheading an initiative that combines data derived from human microphysiological systems (MPS) (i.e., organs on a chip, OoC or tissue chips, $\mathrm{TC}$ ) with in vivo information. Quantitative Systems Pharmacology (QSP) is then used to predict the human clinical effects (toxicity and efficacy) of therapeutic moieties across potential patient populations. These technologies can be used for exploringdrug PK, diseasemodels, drug pharmacodynamic attributes, or even medicines (e.g., biomarker discovery). The kinds of information derived from the use of these tools can vary, depending upon the complexity of the system (e.g., one versus multiple integrated platforms) and the nature of the questions being addressed. These questions typically take on increasing levels of complexity as the drug application moves from the initial stages of rapid high throughput screening to the more targeted questions associated with compound advancement to drug candidate (be that a small molecule, a biologic or a nanoformulation).

An MPS consists of the platform (chip, device), the tissue construct, and the tissue culture media. A determination of optimal application of an MPS depends not only upon the tissue being modeled but also upon the platform properties. For example, non-specific binding (adsorption) of active pharmaceutical ingredient depends on platform material. Since the various platform materials (polydimethylsiloxane, polystyrene, polycarbonate, polysulphone) can have different adsorptive properties, there

Dissolution 
is a need to characterize the API-platform adsorption characteristics. Another consideration is the binding of drugs to plasma proteins since either human or bovine serum albumin is contained in most cell culture media, which is essential for an IVIVC as it pertains to the drug properties.

The utility of this tool has been demonstrated by recent contributions such as:

- Integration with nonlinear mixed effect (NLME) models to predict population variability in hepatic drug metabolism (1). Cryopreserved hepatocytes from five different donors were used for evaluating the clearance and metabolism of six compounds. Inter and intra-individual variability was estimated and provided as input for stochastic simulations that successfully predicted the observed clinical concentration-time profiles and the associated population variability.

- Identification of clinically relevant biomarkers to predict early drug-induced liver toxicity in humans (2). The in vitro system consisted of cryopreserved primary human hepatocytes and Kupffer cells that were seeded into a LiverChip bioreactor. The metabolism and clearance of diclofenac, which can induce hepatotoxicity (reduction in function and cell death over the multiple doses, particularly when present in high concentrations) was evaluated in the presence or absence of lipopolysaccharides (LPS). In addition to identifying potential biomarkers of liver toxicity, the study provided insights into the potentiation of toxicity due to the activation of oxidative stress-mediated pathways and altered activity of various CYP and UGT-associated enzymes.

- Use of kidney MPS to identify proximal tubule injury biomarkers (3).

Importantly, no singular organ system can, in isolation, adequately explain drug safety and efficacy (or lack thereof). Therefore, there is an ongoing effort to expand this technology to include multiple integrated platforms. For example, drug characterization is being explored from the perspective of integrated gut and liver MPS systems $(4,5)$. This innovation allows for an investigation of the complex crosstalk that can exist among different organ systems. More recently, there has been an effort to develop and implement multi-MPS platforms (physiome-on-a-chip) that support 4-way, 7-way, and 10way MPS interactions (6). While theoretically attractive, there are technical challenges associated with these multi-MPS platforms: 1) the creation and maintenance of MPSs that exhibit sufficiently representative and robust physiological function over extended culture periods typically require procurement and preparation of primary cells or pluripotent-stem cells to reach functional maturity in specialized microenvironments; 2 ) there is the need to design and produce platform hardware that can accommodate and sustain the relevant MPSs. This can be difficult, considering the differences in system maturation times and media; 3 ) selection of a medium composition compatible with the different MPSs on the platform; and 4) practical and translational considerations such as flow partitioning, flow rates, physiological sensors, sampling frequency, and sample volume

While more work is needed before these systems are readily adopted into drug development and assessment programs, it is clear that the information that can be derived from the MPS platform will be invaluable in terms of time efficiency, specificity (ability to isolate and explore specific questions in a well-controlled environment), and ability to inform in silico models in ways that may not have been possible through traditional clinical or preclinical studies.

\section{REFERENCES}

1. Tsamandouras, N.; Kostrzewski, T.; Stokes, C. L.; Griffith, L. G.; Hughes, D. J.; Cirit, M. Quantitative assessment of population variability in hepatic drug metabolism using a perfused three-dimensional human liver microphysiological system. J. Pharmacol. Exp. Ther. 2017, 360, 95-105. DOI: 10.1124/ jpet.116.237495.

2. Sarkar, U.; Ravindra, K. C.; Large, E.; Young, C. L.; Rivera-Burgos, D.; Yu, J.; Cirit, M.; Hughes, D. J.; Wishnok, J. S.; Lauffenburger, D. A.; Griffith, L. G.; Tannenbaum, S. R. Integrated assessment of diclofenac biotransformation, pharmacokinetics, and omics-based toxicity in a three-dimensional human liverimmunocompetent coculture system. Drug Metab. Dispos. 2017, 45, 855-866. DOI: 10.1124/dmd.116.074005.

3. Maass, C.; Sorensen, N. B.; Himmelfarb, J.; Kelly, E. J.; Stokes, C. L.; Cirit, M. Translational assessment of drug-induced proximal tubule injury using a kidney microphysiological system. CPT Pharmacometrics Syst. Pharmacol. 2019, 8, 316-325. DOI: 10.1002/psp4.12400.

4. Tsamandouras, N.; Chen, W. L. K.; Edington, C. D.; Stokes, C. L.; Griffith, L. G.; Cirit, M. Integrated gut and liver microphysiological systems for quantitative in vitro pharmacokinetic studies. AAPS J. 2017, 19, 14991512. DOI: 10.1208/s12248-017-0122-4.

5. Chen, W. L. K.; Edington, C.; Suter, E.; Yu, J.; Velazquez, J. J.; 
Velazquez, J. G.; Shockley, M.; Large, E. M.; Venkataramanan, R.; Hughes, D. J.; Stokes, C. L.; Trumper, D. L.; Carrier, R. L.; Cirit, M.; Griffith, L. G.; Lauffenburger, D. A. Integrated gut/ liver microphysiological systems elucidates inflammatory intertissue crosstalk. Biotechnol. Bioeng. 2017, 114, 2648-2659. DOI: 10.1002/bit.26370.

6. Edington, C. D.; Chen, W. L. K.; Geishecker, E.; Kassis, T.; Soenksen, L. R.; Bhushan, B. M.; Freake, D.; Kirschner, J.; Maass, C.; Tsamandouras, N.; Valdez, J.; Cook, C. D.; Parent, T,; Snyder, S.; Yu, J.; Suter, E.; Shockley, M.; Velazquez, J.; Velazquez, J.J.; Stockdale, L.; Papps J. P.; Lee, I.; Vann, N.; Gamboa, M.; LaBarge, M. E.; Zhong, Z.; Wang, X.; Boyer, L. A.; Lauffenburger, D. A.; Carrier, R. L.; Communal, C.; Tannenbaum, S. R.; Stokes, C. L.; Hughes, D. J.; Rohatgi, G.; Trumper, D. L.; Cirit, M.; Griffith, L. G. Interconnected microphysiological systems for quantitative biology and pharmacology studies. Sci Rep. 2018, 8, 4530. DOI: 10.1038/s41598-018-22749-0.

\section{DRUG PRODUCT CHARACTERIZATION AS A MECHANISM FOR SUPPORTING BIOWAIVERS Mansoor A. Khan}

Rangel College of Pharmacy, Texas A\&M University, College Station, TX, USA.

Bioavailability studies are typically done as benchmarking tests to evaluate the rate and extent of drug absorption via measurements of peak drug concentrations $\left(C_{\max }\right)$ and extent of exposure, as described by an area under the concentration versus time profile (AUC). If a compound has less bioavailability with respect to the extent of absorption, it can be enhanced using a variety of approaches. These include excipient selection, formulations, or changing the method of drug delivery (including changes in route). In contrast, BE tests are a relative measure for determining the degree of sameness to a reference product. As an example, a generic product is required to show that it is pharmaceutically equivalent and bioequivalent to be substitutable as a therapeutically equivalent to the brand product. Other areas where $\mathrm{BE}$ needs to be demonstrated include bridging studies where quality related changes are made after approval of some PK or clinical studies. Examples of these changes are included in FDA's scale-up and post approval changes (SUPAC) guidance documents, particularly in the case of level three changes where there are alterations made in product formulation, processes, or site change (unless a biowaiver is granted). These BE trials help avoid the need to repeat clinical studies.

The granting of biowaivers implies that the demonstration of in vivo BE can be based on the use of surrogate tests. In addition to waiver of an in vivo $B E$ requirement under 21 CFR 320.22, there are circumstances in which BE can be evaluated using in vitro approaches under 21 CFR320.24(b) (6). The scientific principles described in the guidance regarding waiver of an in vivo requirement also apply to consideration of in vitro data under 21 CFR 320.24(b)(6). In such circumstances, an in vivo data requirement is not waived, but rather, FDA has determined that in vitro is the most accurate, sensitive, and reproducible for a product, as required under 21 CFR 320.24(a).

Biowaivers can be granted for parenteral solutions where excipients are qualitatively (Q1) and quantitatively (Q2) same, or for inhalation products where the drug is delivered as a gas and the API and dosage forms are same between the brand and the generic products. Biowaivers are also granted for oral solutions, syrups, elixirs, tinctures, nebulizers, nasal solutions, topical solutions, that has same drug, same concentration, and same dosage form, and same route as the RLD, and contains no excipient that significantly affect absorption or local availability. Additionally, biowaivers may be granted for BCS Class 1 compounds (High solubility and high permeability of $\mathrm{API}$, and rapidly dissolving drug product), BCS Class 3 compounds (High solubility and poor permeability drugs when the drug product is rapidly dissolving, qualitatively same and quantitatively similar to the reference as defined by the criteria specified by SUPAC levels 1 and 2). Biowaivers are also granted when IVIVC relationships are known, or when products of multiple strengths are developed, and BE is evaluated on higher strength dosage forms in many cases.

Despite our current knowledge and experience, there remains numerous products where the determination product BE is contentious. This difficulty has given rise to the new topic of "complex generics" where there are no direct means of determining BE by traditional approaches of plasma or body fluid concentration measurements. For example, when oral products are developed for local action within the GI tract, there is no or negligible absorption into the systemic circulation. In such situations, the question remains as to how to determine product BE. In 21 CFR 320.1, bioequivalence is defined as "The absence of a significant difference in the rate and extent to which the active ingredient or active moiety in pharmaceutical equivalents or pharmaceutical alternatives becomes available at the site of drug action when administrated at the same molar dose under similar conditions in an appropriately designed study..." Fortunately, there are other 21 CFR regulations that allow for $\mathrm{BE}$ determinations by non-traditional approaches. 
In 21 CFR 320.14, BE can be determined by in vivo measurement of active moiety or moieties in biologic fluid $O R$ in vivo pharmacodynamic comparison $O R$ by with a "bioequivalence study with clinical endpoints" OR an in vitro comparison OR any other approach deemed appropriate by FDA.

The presentation included numerous practical cases of $\mathrm{BE}$ determination of complex generics. As an example, vancomycin is used for enteric infections due to clostridium difficile but does not get systemically absorbed. Despite protests by the brand firm, FDA decided to use in vitro dissolution approaches for sameness determination, and approved generic products based on this in vitro approach. As indicated in Figure 1, vancomycin dissolves faster in the lower $\mathrm{pH}$ as compared to higher $\mathrm{pH}$. Since the $\mathrm{pH}$ media of patients may vary, it was decided to compare the brand and generic products in multiple $\mathrm{pH}$ media, including biorelevant (BR) media at pH 4.5 and 6.8 .

Similarly, lanthanum carbonate is used locally in the GI tract for hyperphosphatemia. It acts by binding to dietary phosphates in end stage renal disease. In this case, sameness can be determined by comparing product drug

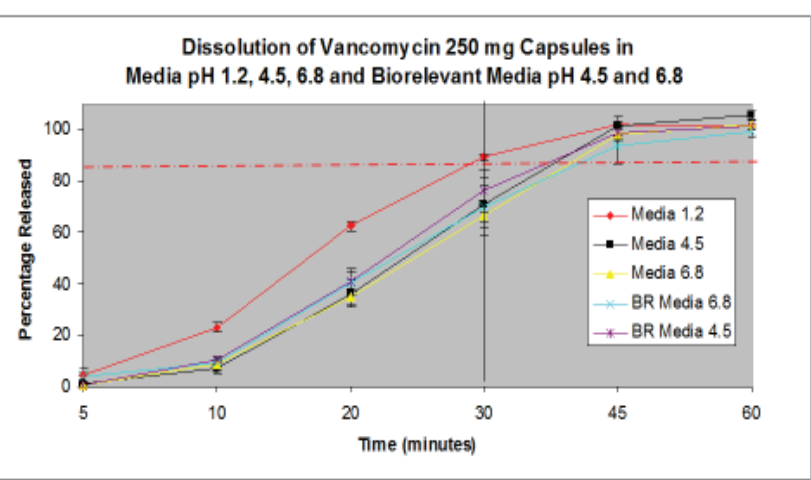

Figure 1. Influence of $\mathrm{pH}$ on vancomycin in vitro dissolution profiles. Source: Internal study, United Stated Food and Drug Administration.

release across several in vitro media (varying the $\mathrm{pH}$ ), as well as the rate and extent of binding of lanthanum carbonate phosphates by kinetic and equilibrium binding studies (1). Figure 2 illustrates the closeness of certain generic products to the brand at low and high levels of phosphates in $\mathrm{pH} 1.2$ and 4.5. Accordingly, the kinetic as well as equilibrium binding constants show similarity between the brand and generic products.

Other cases that were highlighted for BE determination of complex generics included colloidal iron, acyclovir ocular
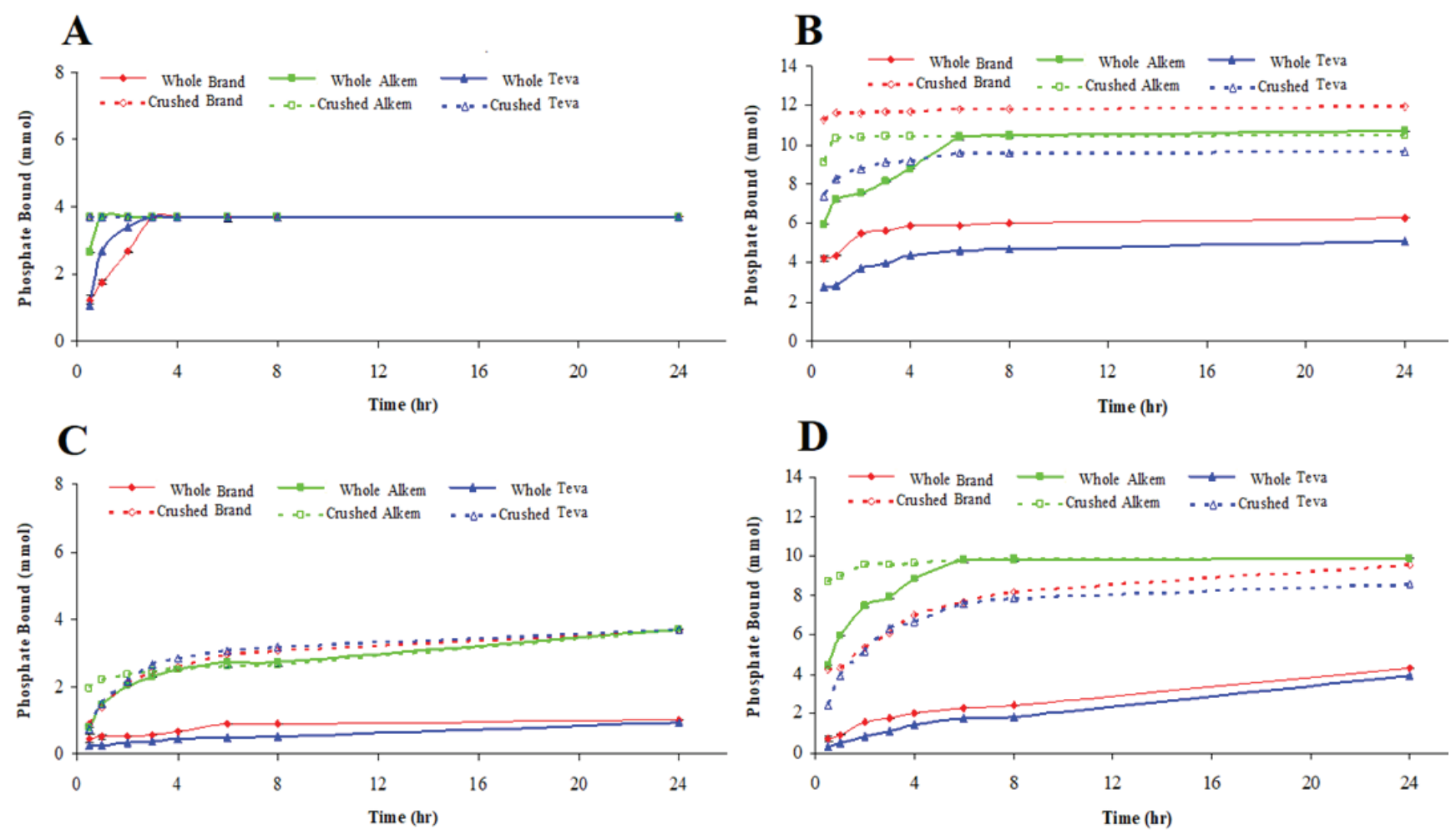

Figure 2. A-D: Influence of $\mathrm{pH}$ on the phosphate binding of three brands (Brand [red], Alkem [green], Teva [blue]) of lanthanum carbonate phosphate tablets (whole [solid line] and crushed [dashed line] tablets) under conditions of high ( $B$ and $D$ ) versus low ( $A$ and $C$ ) phosphate concentrations. Source: Internal study, United States Food and Drug Administration. 
ointment $(2,3)$, acyclovir skin creams and transdermal products (4).

\section{REFERENCES}

1. Yang, Y.; Shah, R. B.; Yu, L. X.; Khan, M. A. In vitro bioequivalence approach for a locally acting gastrointestinal drug: lanthanum carbonate. Mol. Pharm. 2013, 10, 544-550. DOI: 10.1021/ mp300517p.

2. Al-Ghabeish, M.; Xu, L. X.; Krishnaiah, Y.; Rahman, Z.; Yang, Y.; Khan, M. Influence of drug loading and type of ointment base on the in vitro performance of acyclovir ophthalmic ointment. Int. J. Pharm. 2015, 495, 783-791. DOI: 10.1016/j. ijpharm.2015.08.096.

3. Xu, X.; Al-Ghabeish, M.; Rahman, Z.; Krishnaiah, Y. S.; Yerlikaya, F.; Yang, Y.; Khan, M. A. Formulation and process factors influencing product quality and in vitro performance of ophthalmic ointments. Int. J. Pharm. 2015, 493, 412-425. DOI: 10.1016/j. ijpharm.2015.07.066.

4. Krishnaiah, Y. S.; Xu, X.; Rahman, Z.; Yang, Y.; Katragadda, U.; Lionberger, R.; Peters, J. R.; Uhl, K.; Khan, M. A. Development of performance matrix for generic product equivalence of acyclovir topical creams. Int. J. Pharm. 2014, 475, 110-122. DOI: 10.1016/j.ijpharm.2014.07.034.

\section{QUESTIONS AND ANSWERS}

\section{IN VITRO DISSOLUTION AND FORMULATION PERFORMANCE \\ "Characterization of GI Fluids Content, Viscosity, Vol- ume in Dogs and Humans: Comparison Under Fasted and Fed Conditions" by Christos Reppas, PhD, Department of Pharmacy, National and Kapodistrian University of Athens, Athens, Greece}

\section{"Examples of Dissolution Differences When Using Canine vs Human Biorelevant Media: Working to Op- timize In Vitro Methods That Support the Translation of In Vivo Oral Drug Product Dissolution in Dogs vs Humans" by Maria Vertzoni, PhD, Department of Pharmacy, National and Kapodistrian University of Athens, Athens, Greece}

Q: Is there an advantage to using biorelevant media when trying to generate an IVIVC?

A: As part of efforts to generate an IVIVC (or to establish QC test conditions), it is important to explore a range of in vitro media. The most appropriate will be a function of the physicochemical characteristics of the API and the CQA of the formulation(s) in question. While typically, it would seem logical for the biorelevant media to be the most informative, it is important to recognize that conditions within the Gl tract vary as a function of intestinal segment. Moreover, the in vitro conditions most predictive of in vivo product performance is linked to the formulation CQA. Thus, the IVIVC needs to incorporate product understanding.
Q: How does one define the discriminatory power of the dissolution test?

A: The dissolution method should be able to identify any change during the manufacturing that affects the in vivo performance.

Q: Why is it necessary to maintain a constant $\mathrm{pH}$ from the beginning to the end of a dissolution run?

A: Any change in $\mathrm{pH}$ would affect the solubility (amount of release) and the product performance. Given that traditional in vitro dissolution test conditions are not intended to replicate the dynamics of the Gl tract, it is necessary to have enough buffer capacity to maintain stable, well-controlled conditions.

Q: Is it necessary for the in vitro media to be biorelevant?

A: An in vitro performance test is termed biorelevant when it mimics intraluminal performance of the dosage form. Depending on the API and the product, the intraluminal performance, could be mimicked with a simple aqueous buffer. In such case the buffer system is a biorelevant medium. The in vitro media should in all cases be biorelevant.

\section{CHARACTERIZATION OF API SOLUBILITY, PER-}

MEABILITY, AND DRUG METABOLISM

"ECCS as a Tool for Facilitating Interspecies Extrapolation" by Ayman El-Kattan, B. Pharm., PhD, DMPK Head, IFM Therapeutics, Boston, MA, USA.

“Use of Flux Measurements in Lieu of In Vitro Dissolution to Assess the Complex Interplay Between Solubility, Permeability and Formulation Effects" by Konstantin Tsinman, PhD, Chief Scientific Officer, Pion, Inc. Billerica, MA, USA.

“Use of Dogs to Support Formulation Development for Large Molecule Oral Drug Delivery" by Patrick Sinko, $P h D, R P h$, Associate Vice President, Office of Research Advancement, Rutgers University, Piscataway, NJ, USA.

“Comparison of Effective Permeability Estimating Using Data Generated in Using Chamber (human, dog, rat), MDCK Cells and Caco-2" by Sid Bhoopathy, PhD, Chief Operating Officer, Absorption Systems, Exton, PA, USA.

\section{"Canine Organoids for Drug Permeability Testing:} Moving Beyond Caco-2 Cell Systems" by Jonathan Mochel, DVM, MSc, PhD, Associate Professor, College of Veterinary Medicine, lowa State University, Ames, IA, USA.

Q: What benefits are associated with use of an organoid system that is not currently available when using other systems such as Caco-2 cells??

A: Unlike the Caco-2 systems, the organoids reflect the differences in drug absorption and metabolism associated with disease. Since organoids can be generated from stem cells harvested from healthy and diseased intestines, they uniquely address the impact of disease on drug permeability 
and/or enterocyte metabolism. Colonoids are highly relevant for studying the effects of Crohn's and ulcerative colitis.

\section{“Use of the Dog to Predict Supersaturation and Its Impact on Drug Absorption" by Sara Carlert, PhD, Senior Scientist Biopharmaceutics, AstraZeneca, Gothenburg, Sweden.}

Q: While in vitro systems provide an understanding of precipitating candidate molecules, they often exaggerate the magnitude of precipitation that occurs within the Gl tract. Given this in vivo/in vitro inconsistency, if we rely on in vitro data as the basis for our calculation of in vivo absorption characteristics we're going to overestimate the in vivo absorption potential. With that in mind, how can a sponsor bridge these two discordant sets of observations?

A: To develop informative in vitro and in silico models and to validate these systems, it is necessary to have in vivo data with to ground the evaluation of formulation challenges using these alternative systems.

Q: How often do false negatives lead to eliminating compounds that would have been worthy of further development?

A: This question highlights the importance of not discarding potential drug candidates due simply to its propensity to precipitate in vitro. Moreover, there are numerous formulation options that can be used to reduce this in vivo risk.

Q: How can we predict in vivo precipitation when we don't know how much of the drug is going to be passing from the stomach into the small intestine at any given point of time?

A: It is a misconception to believe that we don't know what will be leaving the stomach. Since the entire dose enters the stomach and none of that drug is absorbed before entering the small intestine, we know that all of the administered dose will eventually exit into the Gl tract. However, what is not known is the exact concentration of drug in the small intestine at any point in time. For that reason, it is important to consider a range of concentrations.

\section{“Drug Product Characterization as a Mechanism for Supporting Biowaivers" by Mansoor Khan, PhD, Professor \& Vice Dean, Rangel College of Pharmacy, Texas A\&M Univer- sity, College Station, TX, USA.}

Q: If an innovative product is associated with marked interlot variability in its in vitro dissolution characteristics (irrespective of medium used), how will this influence the constraints placed on a generic formulation?

A: For complex products, the advice would be to examine multiple lots of the innovator formulation to understand its variability (it may require a six or more reference lots, depending on the magnitude of the variability. The variability of the generic formulation should be contained within the range of the reference product in vitro dissolution profiles. Generic product QC specifications should be set to ensure that this constraint is maintained as a pre-requisite for batch release

Q: At the USP we're looking at developing in vitro methods for testing the performance of novel or special dosage forms. Is the FDA or industry considering the use of in vitro dissolution testing or in vitro release testing (all types of products - ocular, inhalation products) for evaluating product $\mathrm{BE}$ ?

A: There is no singular opinion on the applicability of these in vitro methods for evaluating product BE. There are published examples of dissolution test conditions that fail to discriminate between inequivalent formulations while other methods can detect these differences. The other point to consider is the purpose of the test. When used for batch release, the assumption is that the CQA is well defined by the in vitro test method and that any dispersion outside of the QC limits imply a potential change that can influence in vivo product performance. On the other hand, the same method may not be capable of identifying formulation changes that can lead to a change in the CQA. For that reason, the test method appropriate for batch release may not be likewise appropriate for supporting a change in product formulation or manufacturing method. Moreover, sometimes there is a need to match the method with the product. For example, considering products with a matrix composed of HPMC, some methods may fail to adequately identify the dose dumping that can occur in vivo for formulations associated with inducing a supersaturated condition where it may be necessary to use non-sink rather than sink conditions for formulation comparison.

\section{FORMULATION CONSIDERATIONS: BEYOND IN}

VITRO DISSOLUTION

"In Vivo Excipient Effects Not Predicted In Vitro" by

Talia Flanagan, PhD, Previously: Associate Principal Scientist Biopharmaceutics, Research and Development, AstraZeneca, Macclesfield, Cheshire, UK; Present: UCB Pharma SA, Product Development, Chemin du Foriest, Belgium.

"Characterization of a Novel Cross-Linked Lipase: Impact of Cross-Linking on Solubility and Release from Drug Product" by David Sperry, Research Advisor, Small Molecule Design \& Development, Eli Lilly and Company, Indianapolis, IN, USA.

"Product Understanding and DOE as a Mechanism for Developing Dissolution Method" by Raafat Fahmy, PhD, Science Advisor for Chemistry and Manufacturing Issues, U.S. FDA, Center for Veterinary Medicine, Rockville, MD, USA.

“Using In Vitro Tools to Predict Pulmonary Drug Delivery" by Ben Forbes, PhD, Professor, Institute of Pharmaceutical Sciences, Kings College London, London, UK.

Q: Is there the potential for dissolution-rate limited drug absorption in the lung?

A: Drugs are absorbed very rapidly from the lungs and the vast majority of APIs are not dissolution limited. This is both because of the physicochemical properties of the API's 
targeted for pulmonary delivery. Typically, the particles are extremely small ( 2 microns or smaller) and their deposition within a phospholipid-rich area further facilitates the in vivo dissolution process.

"Case Studies From Eye, Skin and GI Tract" by Mansoor Khan, PhD, Professor and Vice Dean, Rangel College of Pharmacy, Texas A\&M University, College Station, TX, USA.

Q: Some ointments have a wide range of viscosities (10K $90 \mathrm{~K}$ cps). However, for launch within the EU, it is considered unacceptable to approve a product with such a wide range of viscosity. As a result, there is a problem with failed lots that cannot be marketed. What kind of justification would be needed other than modification of the manufacturing method to allow for a wider specification range for product viscosity

A: Assuming that an appropriate method is being used for evaluating product viscosity, then specifications should be based upon the viscosities demonstrated to be effective pre-approval. Post-approval lots should remain within those specifications. If that cannot be achieved, additional in vivo data may be needed.

\section{IN SILICO MODELS}

"In Silico Modeling of Oral Drug Absorption in Dogs vs Humans: Differences in GI Tract Physiology of Humans and Dogs (intestinal transporters, gut metabolism, absorptive surface area)" by Devendra Pade, PhD, Senior Research Scientist, Certara UK Limited (Simcyp Division), Blades Enterprise Center, Sheffield, UK.

\section{"Use of In Silico Mechanistic Models to Support Inter- species Extrapolation of Oral Bioavailability and For- mulation Optimization: Model Example Using Gastro- Plus" by Viera Lukacova, PhD, Director, Simulation Sciences, Simulations Plus, Inc., Lancaster, CA, USA.}

Q: When considering the area available for absorption, why would it be considered differently (based upon the use of an area extension factor) for lipophilic versus hydrophilic drugs? Isn't the villus surface area a physiological constant?

A: While the physiological villus surface area is constant for each individual, the model needs to have the flexibility to adjust for the portion of that total surface areas associated with the drug absorption process. For that, the intrinsic permeability of a drug reflects its ability to move across the biological membrane, which is a function of its hydrophilicity or lipophilicity. Compounds that are highly permeable (have the lipophilicity needed to cross the biological membrane), typically occupy only the villus tip. While lower permeability typically occupies the villus entire surface area. The extension factor provides model flexibility to adapt the model as a function of drug physicochemical properties.

Q: Models are based upon data generated in subjects (humans and dogs) that have a healthy intestine: Clinically, we typically see all kinds of physiological changes, from microbial imbalances (which leads to altered luminal metabolism and altered bile salt conjugation) to a patchy loss of surface area.
How can a PBPK model help predict in vivo drug absorption for the human or canine patient?

A: Models can be adjusted to accommodate the physiological changes occurring with disease. However, data are needed to inform the model so that they can be refined to reflect specific disease states.

Q: If your model prediction fails, how do you know if the problem is with the model itself or the input data?

A: It depends on the specific situation. When appropriate, we consider if a different mechanism would provide a better explanation of the observed in vivo data. The other critical point is that within the context of the model, we need to consider what constitutes the more influential variables and the likely corresponding range of parameter values that would be consistent with the observations (sensitivity analysis). For example, the effect of formulation for a compound with a distinct absorption window (such as ciprofloxacin), was more pronounced in dogs than humans. However, if a drug is well absorbed throughout the Gl tract, intestinal transit time would be expected to have far less impact so that interspecies differences in $\mathrm{Gl}$ transit time be negligible unless formulated so that dissolution rate becomes a rate-limiting factor.

Q: Given that there are numerous interacting factors influencing in vivo product performance, how can this 3D perspective be incorporated into the in-silico sensitivity analysis?

A: Based upon the model assumptions, the sensitivity analysis provides an assessment of how output parameter values will change as a function of the value of the input parameters. A single factor or multiple input parameters can be varied the most influential parameters/effect of a range of parameter values can be assessed relative to the range of values associated with the other parameters included in the analysis.

\section{FINAL TAKE-HOME MESSAGES}

Powerful tools are available to support product formulation optimization, lead candidate selection, and refinement of dosage regimens to address the many potential sources of variability in the dose-exposure response relationships. However, it is essential that the strengths and limitations of these methods be fully appreciated. For example, regarding in vitro dissolution, several key points need to be integrated into the study design and corresponding data interpretation: 1) the purpose of the test (QC testing, formulation optimization, BE evaluation, specification establishment); 2) the biorelevance of the media and test conditions; 3 ) the CQA of the formulation in question; and 4) the physicochemical characteristics of the API.

To identify an in vitro dissolution test that is appropriately discriminating, clinical relevance via in vivo studies, supplemented by in silico assessments is an important 
part of method development. Regarding the utility of PBPK simulations, the first two critical steps involve defining its purpose (e.g., predict API druggability, formulation optimization, and predictions of product performance in the target patient population) and establishing a protocol for model verification. Models can also be used to explore the relative importance of errors that may exist across the input parameters and to optimize the efficiency of the experimental roadmap used in support of product development.

When asked about what they considered to be the points of emerging impact coming in their respective fields over the next five years, the following comments were shared.

- Patrick Sinko: The fact that we're so focused on delivering the API. Nano allows us to think of it differently, ferrying the particles across.

- Christos Reppas: Oral absorption in humans colonic absorption?

- Sid Bhoopathy: Dissolution, permeability - can we combine that into an apparatus?

- Viera Lukacova: From a modeling standpoint applications of the modeling tools, validation will increase confidence.

- Sara Carlert: Precipitation and supersaturation - my dream is that in 5-10 years we'll be able to quantitatively predict.
- Konstantin Tsinman: My thoughts are similar to Dr Reppas. In view of properties in vitro setup will increase.

- Maria Vertzoni: Increase knowledge of differences along the Gl tract for special populations.

As we move toward a goal of individualized patient care, in-hospital use of PBPK models, genetic testing, functional imaging (e.g., blood flow around a tumor) and 3D printing may eventually lead to the availability of patient-specific medications and dosage regimens. Such changes in patient care would impact the scientific needs and critical questions to be considered by drug sponsors, regulators, and physicians (or veterinarians). This futuristic vision may well be within the trajectory of advances emerging among our community of pharmaceutical scientists.

\section{ACKNOWLEDGEMENTS}

The authors of this workshop report express our sincere thanks to Dr. Margareth Marques (Principal Scientific Liaison) and Ms. Renee Stake (Manager, Meetings \& Marketing) without whose invaluable support and input this workshop would not have been possible. We also express our thanks to Ms. Christina Chase (Technical Writer IV), whose summary of presentations subsequent discussions provided the framework for this meeting report. 